\title{
Leptogenesis in $E_{6} \times \mathrm{U}(1)_{A}$ SUSY GUT model
}

\author{
Takuya Ishihara, ${ }^{a}$ Nobuhiro Maekawa, ${ }^{a, b}$ Mao Takegawa ${ }^{a}$ and Masato Yamanaka ${ }^{b}$ \\ ${ }^{a}$ Department of Physics, Nagoya University, \\ Nagoya 464-8602, Japan \\ ${ }^{b}$ Kobayashi Maskawa Institute, Nagoya University, \\ Nagoya 464-8602, Japan \\ E-mail: ishihara@eken.phys.nagoya-u.ac.jp, \\ maekawa@eken.phys.nagoya-u.ac.jp, takegawa.mao@kao.co.jp, \\ yamanaka@eken.phys.nagoya-u.ac.jp
}

AbStract: We study the thermal leptogenesis in the $E_{6} \times \mathrm{U}(1)_{A}$ SUSY GUT model in which realistic masses and mixings of quarks and leptons can be realized. We show that the sufficient baryon number can be produced by the leptogenesis in the model, in which the mass parameter of the lightest right-handed neutrino is predicted to be smaller than $10^{8} \mathrm{GeV}$. The essential point is that the mass of the lightest right-handed neutrino can be enhanced in the model because it has a lot of mass terms whose mass parameters are predicted to be the same order of magnitude which is smaller than $10^{8} \mathrm{GeV}$. We show that $\mathrm{O}(10)$ enhancement for the lightest right-handed neutrino mass is sufficient for the observed baryon asymmetry. Note that such mass enhancements do not change the predictions of neutrino masses and mixings at the low energy scale in the $E_{6}$ model which has six righthanded neutrinos. In the calculation, we include the effects of supersymmetry and flavor in final states of the right-handed neutrino decay. We show that the effect of supersymmetry is quite important even in the strong washout regime when the effect of flavor is included. This is because the washout effects on the asymmetries both of the muon and the electron become weaker than that of the tau asymmetry.

Keywords: Supersymmetry Phenomenology

ArXiv EPrint: 1508.06212 


\section{Contents}

1 Introduction 1

$2 \quad E_{6}$ unification with anomalous $U(1)_{A}$ gauge symmetry 4

$\begin{array}{lll}3 & \text { Possible enhancement for the right-handed neutrino masses } & 7\end{array}$

4 Leptogenesis in the $E_{6} \times \mathrm{U}(1)_{A}$ model $\quad 8$

4.1 non-SUSY + non-flavor $\quad 9$

$\begin{array}{llr}4.2 & \text { Non-SUSY + flavor } & 12\end{array}$

$\begin{array}{lll}4.3 & \text { SUSY + non-flavor } & 15\end{array}$

$\begin{array}{lll}4.4 & \text { SUSY + flavor } & 16\end{array}$

$\begin{array}{llr}5 & \text { Other baryogenesis } & 19\end{array}$

6 Summary and discussion $\quad 20$

\section{Introduction}

Supersymmetric (SUSY) grand unified theory (GUT) [1-5] is one of the most promising candidates as the extended model of the standard model (SM). This is because the SUSY GUT realizes two kinds of unifications, unification of the gauge interactions and unification of the matters in the SM and for both unifications, there are supports from experiments. Three gauge couplings in the SM meets at a scale, which is called the GUT scale $\Lambda_{G} \sim$ $2 \times 10^{16} \mathrm{GeV}$. Moreover, the various hierarchies of quark and lepton masses and mixings can be naturally understood in SU(5) unification if we assume that the $\mathbf{1 0}$ fields of SU(5) induce stronger hierarchy in Yukawa couplings than the $\overline{\mathbf{5}}$ fields of SU(5). One of the most important advantages of the $E_{6}$ unification [6-13] is that the above assumption can be naturally derived [14]. As the result of this important feature of the $E_{6}$ unification, we can build an $E_{6}$ GUT in which all three generation of quarks and leptons can be unified into a single multiplet(or two multiplets) by introducing family symmetry $\mathrm{SU}(3)_{F}$ (or $\mathrm{SU}(2)_{F}$ ) and the realistic quark and lepton masses and mixings can be realized after breaking the family and GUT symmetries [15-20].

However, it is well-known that SUSY GUTs are suffering from the doublet-triplet splitting problem [21]. The doublet Higgs must have the weak scale mass to obtain the weak scale, while the triplet (colored) Higgs which belongs to the same multiplet as the doublet Higgs in the GUT must have the GUT scale mass to stabilize the nucleon. Fortunately, if the anomalous $\mathrm{U}(1)_{A}$ gauge symmetry [22-25] is introduced, the problem can be solved under a natural assumption that all the interactions are introduced with $O(1)$ 
coefficients [26-30]. Because of this natural assumption, the coefficients of the terms and the vacuum expectation values (VEVs) of the GUT Higgs can be determined only by the symmetry of the theory. The coefficients of the interaction $X Y Z$ are determined [31,32] except the $O(1)$ coefficients by the total anomalous $\mathrm{U}(1)_{A}$ charge $x+y+z$ as

$$
\begin{array}{cc}
\lambda^{x+y+z} X Y Z & (x+y+z \geq 0) \\
0 & (x+y+z<0),
\end{array}
$$

where $x, y$, and $z$ are the $\mathrm{U}(1)_{A}$ charges of the fields $X, Y$, and $Z$, respectively. Throughout this paper, we denote all the fields with uppercase letters and their anomalous $\mathrm{U}(1)_{A}$ charges with the corresponding lowercase letters if there is no special comment. Here $\lambda$ is the ratio of the Fayet-Illiopoulos parameter $\xi$ to the cutoff $\Lambda$, and in this paper we take $\lambda \sim 0.22$ as a typical value. ${ }^{1}$ Under the natural assumption, we can obtain the realistic Yukawa couplings in $E_{6}$ GUT [14] (or in SO(10) GUT [26] which has similar structure as $E_{6}$ GUT). The VEVs of the operators $O$ are also determined [14] by their total anomalous $\mathrm{U}(1)_{A}$ charges $o$ as

$$
\langle O\rangle=\left\{\begin{array}{ll}
0 & (o>0) \\
\lambda^{-o} & (o \leq 0)
\end{array} .\right.
$$

In this paper, we often use a unit in which the cutoff $\Lambda$ is taken to be 1 . Because of the natural assumption, all the mass spectrum of superheavy particles and the VEVs of GUT Higgs are determined only by the symmetry of the theory. Therefore, we can calculate the running gauge couplings once we fix the symmetry of the theory. Interestingly, this natural scenario gives a novel explanation $[29,30]$ for the experimental support for the unification of three gauge interactions in the minimal SUSY SM (MSSM). The new explanation requires that the cutoff scale must be taken to be around the usual GUT scale $\Lambda_{G}[29,30]$.

If this natural $E_{6}$ GUT describes our world, it must be consistent with the cosmology. The dark matter can be the lightest supersymmetric particle. In this paper, we discuss the leptogenesis [33] in this scenario. One of the important things in $E_{6}$ unification for the leptogenesis is that the fundamental representation $\mathbf{2 7}$, which is decomposed in the $E_{6} \supset \mathrm{SO}(10) \times \mathrm{U}(1)_{V^{\prime}}$ notation (and in the $\left[\mathrm{SO}(10) \supset \mathrm{SU}(5) \times \mathrm{U}(1)_{V}\right]$ notation) as

$$
\mathbf{2 7}=\mathbf{1 6} \mathbf{6}_{1}\left[\mathbf{1 0}_{1}+\overline{\mathbf{5}}_{-3}+\mathbf{1}_{5}\right]+\mathbf{1 0}_{-2}\left[\mathbf{5}_{-2}+\overline{\mathbf{5}}_{2}^{\prime}\right]+\mathbf{1}_{4}^{\prime}\left[\mathbf{1}_{0}^{\prime}\right]
$$

includes two singlets $S\left(\mathbf{1}^{\prime}\right)$ and $N_{R}^{c}(\mathbf{1})$ under the SM gauge group, which can be the righthanded ( $\mathrm{RH})$ neutrinos. If we introduce three $\mathbf{2 7}$ for three generation quarks and leptons, we have six RH neutrinos. Basically, since the masses and Yukawa couplings of the RH neutrinos are determined by the symmetry, we can examine whether the leptogenesis works well or not in this scenario. Naively, the leptogenesis in this scenario does not work because the lightest $\mathrm{RH}$ neutrino becomes lighter than $10^{8} \mathrm{GeV}$, i.e., this scenario looks not to satisfy the Ibarra's lower bound [34] for the lightest RH neutrino which is $10^{8-9} \mathrm{GeV}$. Actually, in a typical model, the (effective) $\mathrm{U}(1)_{A}$ charges of $S_{i}$ and $N_{R i}^{c}(i=1,2,3)$ are fixed as $\left(\tilde{s}_{1}, \tilde{s}_{2}, \tilde{s}_{3}\right)=(6.5,5.5,3.5)$ and $\left(\tilde{n}_{R 1}^{c}, \tilde{n}_{R 2}^{c}, \tilde{n}_{R 3}^{c}\right)=(6,5,3)$, and therefore, the mass of the

\footnotetext{
${ }^{1}$ Even if we take the different value $\lambda$ from the Cabibbo mixing angle, the results in this paper do not change so much because most of parameters including the $\mathrm{U}(1)_{A}$ charges are fixed by observed values.
} 
lightest RH neutrino $S_{1}$ becomes $M_{S_{1}} \sim \lambda^{13} \Lambda \sim 5.7 \times 10^{7} \mathrm{GeV}$ [14]. Yukawa couplings are also easily estimated because the sum of the (effective) $\mathrm{U}(1)_{A}$ charges of the up-type Higgs $H_{u}$ and doublet-leptons $l_{i}$ become $\left(\tilde{h}_{u}+\tilde{l}_{1}, \tilde{h}_{u}+\tilde{l}_{2}, \tilde{h}_{u}+\tilde{l}_{3}\right)=(0,-0.5,-1)$. The Yukawa couplings among $l_{i}, S_{1}$ and $H_{u}$ become $\left(\lambda^{6.5}, \lambda^{6}, \lambda^{5.5}\right)$. Then, we can estimate two important parameters for the leptogenesis as

$$
\begin{aligned}
K & \equiv \Gamma_{D} / H \sim 40 \\
\epsilon & \equiv \frac{\Gamma\left(S_{1} \rightarrow l+H_{u}\right)-\Gamma\left(S_{1} \rightarrow \bar{l}+H_{u}^{\dagger}\right)}{\Gamma\left(S_{1} \rightarrow l+H_{u}\right)+\Gamma\left(S_{1} \rightarrow \bar{l}+H_{u}^{\dagger}\right)} \sim 5 \times 10^{-9},
\end{aligned}
$$

where $\Gamma_{D}$ and $H$ are the decay width of $S_{1}$ and the Hubble parameter at $T=M_{S_{1}}$, respectively. (In this paper we denote the lepton doublet fields with lowercase letter $l$ in order to avoid the confusion with lepton asymmetry $L$ in the following discussions.) Since the sufficient production of Baryon number requires $K \sim 1$ and $\epsilon \sim 10^{-7}$, this $K$ is too large, and the $\epsilon$ is too small. The produced lepton number is estimated as

$$
Y_{L} \equiv \frac{n_{L}}{s_{0}} \sim 10^{-13}
$$

which is about $O(1000)$ times smaller than the value $Y_{L} \sim 2.5 \times 10^{-10}$ which is required for the sufficient baryon number. Here, $n_{L}$ and $s_{0}$ are the lepton number density and the entropy density today, and for simplicity, we neglect the SUSY contribution, which will be discussed later.

An important observation for leptogenesis in this scenario is that under fixed Yukawa couplings, $K \propto 1 / M_{S_{1}}$ and $\epsilon \propto M_{S_{1}}$. Therefore, larger $M_{S_{1}}$ results in larger baryon number. This observation is critical because in this scenario, the mass of $S_{1}$ tends to be larger than expected one by the symmetry. There are two essential points in this scenario. One of them is that it has a plenty of terms which give mass to $S_{1}$. Each term gives the same order of mass to $S_{1}$ as expected by the symmetry, and the real mass can increase because of the large number of mass terms. The other point is that the predictions for the quark and lepton masses and mixings does not change so much even if the mass of $S_{1}$ becomes larger than expected by the symmetry. This is because the number of RH neutrino flavors becomes larger than three in $E_{6}$ unification. (In $\mathrm{SO}(10)$ unification, it is not avoidable to change the predictions on neutrino sector if one of the RH neutrino masses is taken to be larger than expected one by the symmetry.)

The question is how large enhancement of the mass is needed to obtain the sufficiently large baryon number. It is the main subject in this paper to answer this question.

In section 2, we briefly review the $E_{6}$ GUT with anomalous $\mathrm{U}(1)_{A}$ gauge symmetry. And in section 3, we discuss the enhancement of the RH neutrino masses in this scenario. In section 4 , we would like to answer the above question. First, we calculate the enhancement factor required to obtain the sufficient baryon number with simple non-SUSY Boltzmann equations. Second, we discuss the effect of lepton flavors. Third, the SUSY effect is considered. Finally, we calculate the enhancement factor, including both effects of lepton flavors and of SUSY. We show that only about $\mathrm{O}(10)$ enhancement of the mass of $S_{1}$ is sufficient for the observed baryon number. 


\begin{tabular}{|c|cccccccc|}
\hline & $\Psi_{1}$ & $\Psi_{2}$ & $\Psi_{3}$ & $H$ & $\bar{H}$ & $C$ & $\bar{C}$ & $A$ \\
\hline$E_{6}$ & $\mathbf{2 7}$ & $\mathbf{2 7}$ & $\mathbf{2 7}$ & $\mathbf{2 7}$ & $\overline{\mathbf{2 7}}$ & $\mathbf{2 7}$ & $\overline{\mathbf{2 7}}$ & $\mathbf{7 8}$ \\
$\mathrm{U}(1)_{A}$ & $\frac{9}{2}$ & $\frac{7}{2}$ & $\frac{3}{2}$ & -3 & 1 & -4 & -1 & -1 \\
\hline
\end{tabular}

Table 1. Field contents of matters and GUT Higgs in a typical $E_{6} \times \mathrm{U}(1)_{A}$ GUT [14] and the charge assignment under $E_{6} \times \mathrm{U}(1)_{A}$. Here, $\Psi_{i}(i=1,2,3)$ are three generation quarks and leptons, the VEVs of $H$ and $\bar{H}$ break $E_{6}$ into $\mathrm{SO}(10)$, the VEVs of $C$ and $\bar{C}$ break $\mathrm{SO}(10)$ into $\mathrm{SU}(5)$, and the VEV of $A$ breaks SU(5) into the standard model gauge group. The MSSM Higgs are included in $H$ and $C$.

\section{$2 \quad E_{6}$ unification with anomalous $\mathrm{U}(1)_{A}$ gauge symmetry}

We briefly review the $E_{6} \times \mathrm{U}(1)_{A}$ GUT in this section [14]. The typical quantum numbers of fields in $E_{6} \times \mathrm{U}(1)_{A}$ GUT are shown in table 1. An interesting structure in $E_{6}$ unification is that three of six $\overline{\mathbf{5}}$ of $\mathrm{SU}(5)$ in three matter fields $\Psi_{i}(\mathbf{2 7})$ become superheavy through the Yukawa interactions

$$
\left(Y^{H}\right)_{i j} \Psi_{i} \Psi_{j} H+\left(Y^{C}\right)_{i j} \Psi_{i} \Psi_{j} C
$$

after developing the VEVs $\langle\bar{H} H\rangle \sim \lambda^{-h-\bar{h}}$ and $\langle\bar{C} C\rangle \sim \lambda^{-c-\bar{c}}$, which break $E_{6}$ into $\mathrm{SO}(10)$ and $\mathrm{SO}(10)$ into $\mathrm{SU}(5)$, respectively. Here, the components of Yukawa matrices $Y^{H}$ and $Y^{C}$ are fixed by the total anomalous $\mathrm{U}(1)_{A}$ charges of the corresponding terms $\Psi_{i} \Psi_{j} H$ and $\Psi_{i} \Psi_{j} C$, respectively. $Y^{H}$, which becomes up-type Yukawa matrix because the MSSM Higgs comes mainly from $H$, is taken to be

$$
\left(\begin{array}{ccc}
\lambda^{6} & \lambda^{5} & \lambda^{3} \\
\lambda^{5} & \lambda^{4} & \lambda^{2} \\
\lambda^{3} & \lambda^{2} & 1
\end{array}\right)
$$

except $O(1)$ coefficients in order to obtain the reasonable hierarchical structure of the CKM matrix. Since the Yukawa couplings for $\Psi_{3}$ are larger than those for $\Psi_{2}$ and $\Psi_{1}$ because $\psi_{3} \gg \psi_{1}, \psi_{2}, \overline{\mathbf{5}}_{3}$ and $\overline{\mathbf{5}}_{3}^{\prime}$ become superheavy, and therefore, three light modes $\overline{\mathbf{5}}$ come from the $\Psi_{1}$ and $\Psi_{2}$. This structure naturally explains why 10s of SU(5) induce stronger hierarchy than $\overline{\mathbf{5}} \mathrm{s}$ of $\mathrm{SU}(5)$, which is important to obtain realistic hierarchies of quark and lepton masses and mixings.

The $E_{6} \times \mathrm{U}(1)_{A}$ GUT in table 1 predicts the six RH neutrino masses $M_{\alpha}(\alpha=$ $1,2, \cdots, 6)$ and the Dirac neutrino Yukawa couplings $Y_{\alpha i}(i=1,2,3)$ as in table 2 except $O(1)$ coefficients. In the followings, we briefly review the derivation of these predictions from the model. See refs. $[14,29,30]$ for the detail. The masses of the RH neutrinos can be obtained through the higher dimensional interactions

$$
\left(Y^{\bar{X}} \bar{Y}\right)_{i j} \Psi_{i} \Psi_{j} \bar{X} \bar{Y}, \quad(\bar{X}, \bar{Y}=\bar{H}, \bar{C}),
$$

after developing the VEVs $\langle\bar{H}\rangle \sim \lambda^{-\frac{1}{2}(h+\bar{h})}$ and $\langle\bar{C}\rangle \sim \lambda^{-\frac{1}{2}(c+\bar{c})}$. (These VEVs are determined by the VEV relations for the GUT singlet operators $\bar{H} H$ and $\bar{C} C$ and the $D$ flatness conditions.) For example, the mass of $S_{1}\left(N_{R 1}^{c}\right)$ becomes $\lambda^{2 \psi_{1}+2 \bar{h}-(h+\bar{h})} \Lambda \sim \lambda^{13} \Lambda$ 


\begin{tabular}{|lll|}
\hline Parameter & value & comment \\
\hline$\Lambda_{G}$ & $2.000 \times 10^{16} \mathrm{GeV}$ & GUT scale and the cutoff scale \\
$M_{1}=\lambda^{13} \Lambda_{G}$ & $5.656 \times 10^{7} \mathrm{GeV}$ & 1st RH neutrino mass \\
$M_{2}=\lambda^{12} \Lambda_{G}$ & $2.571 \times 10^{8} \mathrm{GeV}$ & 2nd RH neutrino mass \\
$M_{3}=\lambda^{11} \Lambda_{G}$ & $1.169 \times 10^{9} \mathrm{GeV}$ & 3rd RH neutrino mass \\
$M_{4}=\lambda^{10} \Lambda_{G}$ & $5.312 \times 10^{9} \mathrm{GeV}$ & 4th RH neutrino mass \\
$M_{5}=\lambda^{7} \Lambda_{G}$ & $4.989 \times 10^{11} \mathrm{GeV}$ & 5 th RH neutrino mass \\
$M_{6}=\lambda^{6} \Lambda_{G}$ & $2.268 \times 10^{12} \mathrm{GeV}$ & 6 th RH neutrino mass \\
$Y_{11}=\lambda^{6.5}$ & $5.318 \times 10^{-5}$ & 11 component of $Y_{\nu}$ \\
$Y_{12}=\lambda^{6.0}$ & $1.134 \times 10^{-4}$ & 12 component of $Y_{\nu}$ \\
$Y_{13}=\lambda^{5.5}$ & $2.417 \times 10^{-4}$ & 13 component of $Y_{\nu}$ \\
$Y_{21}=\lambda^{6.0}$ & $1.134 \times 10^{-4}$ & 21 component of $Y_{\nu}$ \\
$Y_{22}=\lambda^{5.5}$ & $2.417 \times 10^{-4}$ & 22 component of $Y_{\nu}$ \\
$Y_{23}=\lambda^{5.0}$ & $5.154 \times 10^{-4}$ & 23 component of $Y_{\nu}$ \\
$Y_{31}=\lambda^{5.5}$ & $2.417 \times 10^{-4}$ & 31 component of $Y_{\nu}$ \\
$Y_{32}=\lambda^{5.0}$ & $5.154 \times 10^{-4}$ & 32 component of $Y_{\nu}$ \\
$Y_{33}=\lambda^{4.5}$ & $1.099 \times 10^{-3}$ & 33 component of $Y_{\nu}$ \\
$Y_{41}=\lambda^{5.0}$ & $5.154 \times 10^{-4}$ & 41 component of $Y_{\nu}$ \\
$Y_{42}=\lambda^{4.5}$ & $1.099 \times 10^{-3}$ & 42 component of $Y_{\nu}$ \\
$Y_{43}=\lambda^{4.0}$ & $2.343 \times 10^{-3}$ & 43 component of $Y_{\nu}$ \\
$Y_{51}=\lambda^{3.5}$ & $4.994 \times 10^{-3}$ & 51 component of $Y_{\nu}$ \\
$Y_{52}=\lambda^{3.0}$ & $1.065 \times 10^{-2}$ & 52 component of $Y_{\nu}$ \\
$Y_{53}=\lambda^{2.5}$ & $2.270 \times 10^{-2}$ & 53 component of $Y_{\nu}$ \\
$Y_{61}=\lambda^{3.0}$ & $1.065 \times 10^{-2}$ & 61 component of $Y_{\nu}$ \\
$Y_{62}=\lambda^{2.5}$ & $2.270 \times 10^{-2}$ & 62 component of $Y_{\nu}$ \\
$Y_{63}=\lambda^{2.0}$ & $4.840 \times 10^{-2}$ & 63 component of $Y_{\nu}$ \\
\hline
\end{tabular}

Table 2. GUT scale $\Lambda_{G}$, Majorana masses of RH neutrinos $M_{\alpha}(\alpha=1,2, \ldots, 6)$, and each component of neutrino Yukawa $Y_{\nu}$ in the $E_{6} \times \mathrm{U}(1)_{A}$ GUT model with $\lambda=0.22$.

$\left(\lambda^{2 \psi_{1}+2 \bar{c}-(c+\bar{c})} \Lambda \sim \lambda^{12} \Lambda\right)$. It is convenient to define the effective $\mathrm{U}(1)_{A}$ charges for any fields $\Psi$ as

$$
\tilde{\psi}=\psi+\frac{1}{5} c_{V}(\Psi)+\frac{1}{2} c_{V^{\prime}}(\Psi)
$$

where $c_{V}(\Psi)$ and $c_{V^{\prime}}(\Psi)$ are the $\mathrm{U}(1)_{V}$ and $\mathrm{U}(1)_{V^{\prime}}$ charges of $\Psi$, respectively. The coefficients in the above equation (2.4) are determined so that the relations $\langle H\rangle \sim \lambda^{-\tilde{h}}$, $\langle\bar{H}\rangle \sim \lambda^{-\tilde{\bar{h}}},\langle C\rangle \sim \lambda^{-\tilde{c}}$, and $\langle\bar{C}\rangle \sim \lambda^{-\tilde{\bar{c}}}$ are satisfied. It is obvious that the relations (1.1) and (1.2) do not change when the effective $\mathrm{U}(1)_{A}$ charges are introduced because the $\mathrm{U}(1)_{V}$ and $\mathrm{U}(1)_{V^{\prime}}$ charges of the $E_{6}$ invariant terms are vanishing. Although special relations between $O(1)$ coefficients due to the $E_{6}$ symmetry (or other original symmetries which are broken in the effective theory) cannot be seen explicitly in the effective model, the effective 
$\mathrm{U}(1)_{A}$ charges are useful to estimate the couplings of any terms allowed by the original symmetry. For example, the Dirac Yukawa couplings are easily estimated by these effective $\mathrm{U}(1)_{A}$ charges as $\left(\lambda^{\tilde{l}_{i}+\tilde{n}_{R j}^{c}+\tilde{h}_{u}}, \lambda^{\tilde{l}_{i}+\tilde{s}_{j}+\tilde{h}_{u}}\right)$. The mass matrices of the RH neutrinos are also calculated as $\left(\begin{array}{cc}\lambda^{\tilde{n}_{R i}^{c}+\tilde{n}_{R j}^{c}} & \lambda^{\tilde{n}_{R i}^{c}+\tilde{s}_{j}} \\ \lambda^{\tilde{s}_{i}+\tilde{n}_{R j}^{c}} & \lambda^{\tilde{s}_{i}+\tilde{s}_{j}}\end{array}\right)$. The RH neutrino masses $M_{\alpha}$ in table 2 can be obtained by diagonalizing the $6 \times 6 \mathrm{RH}$ neutrino mass matrix. In table 2 we change the ordering of the RH neutrinos' generation number $\alpha$ so that smaller number RH neutrino has smaller mass. The Dirac neutrino Yukawa couplings in table 2 use this new index $\alpha$. Even effective higher dimensional interactions which give the light neutrino masses can be estimated as

$$
\lambda^{\tilde{l}_{i}+\tilde{l}_{j}+2 \tilde{h}_{u}} l_{i} l_{j} H_{u}^{2},
$$

which are also derived from the $\mathrm{RH}$ neutrino mass matrix and the Dirac neutrino Yukawa matrix by the seesaw mechanism.

Since the light neutrino masses are not dependent on the RH neutrino's effective U(1) $A$ charges as in eq. (2.5), one may think that the lightest $\mathrm{RH}$ neutrino can be sufficiently heavy by choosing suitable (effective) $\mathrm{U}(1)_{A}$ charges for the RH neutrinos. ${ }^{2}$ However, in $E_{6} \times$ $\mathrm{U}(1)_{A}$ models, the lightest RH neutrino mass, which is expected by the symmetry, cannot be large. Important observation is that the Dirac neutrino Yukawa couplings cannot be large at all because the main component of three light $\overline{5}$ fields $\left(\overline{\mathbf{5}}_{\mathbf{1}}^{\mathbf{0}}, \overline{\mathbf{5}}_{\mathbf{2}}^{\mathbf{0}}, \overline{\mathbf{5}}_{\mathbf{3}}^{\mathbf{0}}\right)$ are $\left(\overline{\mathbf{5}}_{\mathbf{1}}, \overline{\mathbf{5}}_{\mathbf{1}}^{\prime}, \overline{\mathbf{5}}_{\mathbf{2}}\right)$ which come from the 1st two generation fields $\Psi_{1}$ and $\Psi_{2}$. In principle, $\overline{\mathbf{5}}_{\mathbf{2}}^{\mathbf{0}} \sim \overline{\mathbf{5}}_{\mathbf{1}}^{\prime}+\lambda^{\boldsymbol{\Delta}} \overline{\mathbf{5}}_{\mathbf{3}}$ would have large Yukawa couplings if the mixing $\lambda^{\Delta}$ has been $O(1)$. However, it is reasonable to take $\Delta=\psi_{3}+\psi_{1}+\frac{1}{2}(\bar{c}-c+h-\bar{h})$ between 2 and 3, typically $\Delta \sim 2.5$, to obtain bi-large neutrino mixings [14], because the important prediction from the relations $\left(\overline{\mathbf{5}}_{\mathbf{1}}^{\mathbf{0}}, \overline{\mathbf{5}}_{\mathbf{2}}^{\mathbf{0}}, \overline{\mathbf{5}}_{\mathbf{3}}^{\mathbf{0}}\right) \sim\left(\overline{\mathbf{5}}_{\mathbf{1}}, \overline{\mathbf{5}}_{\mathbf{1}}^{\prime}, \overline{\mathbf{5}}_{\mathbf{2}}\right)$, which is $\left(V_{\mathrm{MNS}}\right)_{13} \sim\left(V_{\mathrm{CKM}}\right)_{12}$, is confirmed by recent neutrino experiments [35-39]. Then the explicit Dirac neutrino Yukawa matrix for the RH neutrinos $\left(N_{R i}^{c}\right)$ is given by

$$
Y_{\nu_{D}} \sim\left(\begin{array}{ccc}
\lambda^{6} & \lambda^{\Delta+3} & \lambda^{5} \\
\lambda^{5} & \lambda^{\Delta+2} & \lambda^{4} \\
\lambda^{3} & \lambda^{\Delta} & \lambda^{2}
\end{array}\right) .
$$

The RH neutrino $\left(N_{R 3}^{c}\right)$ mass must be light to obtain the $\tau$ neutrino mass $m_{\nu_{\tau}} \sim 0.05 \mathrm{eV}$, as around $10^{12} \mathrm{GeV}$. Moreover, since the hierarchy of RH neutrino masses are also determined by the $\mathrm{U}(1)_{A}$ charges $\psi_{i}$, the ratio $M_{N_{R 1}^{c}} / M_{N_{R 3}^{c}}$ becomes around $\lambda^{6}$. That makes the mass of the 1st generation RH neutrino $\left(N_{R 1}^{c}\right)$ around $10^{8} \mathrm{GeV}$. Since the another 1st generation $\mathrm{RH}$ neutrino $S_{1}$ which is from singlet under $\mathrm{SO}(10)$ is lighter than $N_{R 1}^{c}$, it is inevitable that the lightest $\mathrm{RH}$ neutrino mass expected by the symmetry becomes smaller than the Ibarra bound $10^{8-9} \mathrm{GeV}$. Such results depend on the experimental inputs, not on the explicit assignment of $\mathrm{U}(1)_{A}$ charges. Therefore, the lightest $\mathrm{RH}$ neutrino mass which is expected by the symmetry cannot be sufficiently large for successful leptogenesis.

One of the most interesting features in the anomalous $\mathrm{U}(1)_{A}$ models is that the higher dimensional interactions give the same contributions to interactions as the lower dimensional interactions. For example, the coefficients of Yukawa interactions $\Psi_{i} \Psi_{j} H$ are deter-

\footnotetext{
${ }^{2}$ Note that the effective $\mathrm{U}(1)_{A}$ charges do not have to respect $E_{6}$ symmetry.
} 
mined by their total $\mathrm{U}(1)_{A}$ charge as $\lambda^{\psi_{i}+\psi_{j}+h}$ except $O(1)$ coefficient. The higher dimensional interactions $\Psi_{i} A \Psi_{j} H$, whose coefficients are also determined by the total charge as $\lambda^{\psi_{i}+\psi_{j}+h+a}$, also contribute to the Yukawa interactions $\Psi_{i} \Psi_{j} H$ after developing the VEV $\langle A\rangle \sim \lambda^{-a}$ which breaks SU(5) into the SM gauge group. The coefficients from the higher dimensional interactions are estimated as $\lambda^{\psi_{i}+\psi_{j}+h+a}\langle A\rangle \sim \lambda^{\psi_{i}+\psi_{j}+h}$, which is nothing but the coefficients of the original Yukawa interactions except $O(1)$ coefficients. Therefore, the unrealistic GUT relations of Yukawa couplings, for example, $Y_{d}=Y_{e}^{t}$, can be naturally avoided in the anomalous $\mathrm{U}(1)_{A}$ GUT models because the higher dimensional interactions with the adjoint Higgs $A$ have different contributions to the down-type Yukawa couplings from the charged lepton Yukawa couplings after developing the VEV of $A$.

\section{Possible enhancement for the right-handed neutrino masses}

It is plausible to enhance a coefficient of an interaction if there are a lot of higher dimensional interactions which contribute to the coefficient by the same order after developing the VEVs of the negatively charged operators. Roughly, if there are $N$ higher dimensional interactions which give the same contribution to an interaction, the enhancement factor can be expected to be $\sqrt{N}$ according to the random walk theory. Since we have introduced several negatively charged singlets as well as the GUT Higgs fields, the number $N$ can be large if the total $\mathrm{U}(1)_{A}$ charge of an interaction is large. For example, in a simplified model in which all negatively charged fields $\Theta_{i}(i=1,2, \cdots, n)$ have the $\mathrm{U}(1)_{A}$ charges $\theta_{i}=-1$, the number of the independent interactions with total $\mathrm{U}(1)_{A}$ charge $c$ is given by $N_{n}(c)=\frac{(n+c-1) !}{c !(n-1) !}$. This number $N_{n}(c)$ becomes easily large when $c$ and $n$ are large. For example, we obtain that $N_{5}(5)=126, N_{5}(10)=1001, N_{10}(10)=92378, \cdots$. In this section, we will show it is plausible that the 1st, 2nd and 3rd smallest RH neutrino masses are enhanced and this enhancement does not change the physical predictions for the light neutrino sector so much.

The interactions which contribute to the masses of the $\mathrm{RH}$ neutrinos $S_{i}$ and $N_{R i}^{c}$ $(i=1,2,3)$ are $\Psi_{i} \Psi_{i} \bar{H} \bar{H}$ and $\Psi_{i} \Psi_{i} \bar{C} \bar{C}$, respectively. The total $\mathrm{U}(1)_{A}$ charges of these interactions are $(11,9,5)$ for $S_{i}$ and $(9,7,3)$ for $N_{R i}^{c}$, while the masses expected by the symmetry are $\left(\lambda^{13}, \lambda^{11}, \lambda^{7}\right)$ and $\left(\lambda^{12}, \lambda^{10}, \lambda^{6}\right)$, respectively. This means that the enhancement factors $\eta_{S_{i}}$ and $\eta_{N_{R i}^{c}}$ for their masses are expected to be the largest for the lightest RH neutrino $S_{1}$, the second largest for the second and the third lightest neutrinos $N_{R 1}^{c}$ and $S_{2}$.

In this paper, we do not count the total number of the independent interactions which give the mass term of these RH neutrinos in the explicit $E_{6}$ GUT model in table 1. However, we discuss what happens when some of the $\mathrm{RH}$ neutrinos have larger masses than those expected by the symmetry. It is an important observation that each $\mathrm{RH}$ neutrino gives the same order of the contribution to all components of the light neutrinos' mass matrix $M_{\nu}=Y_{\nu_{D}}^{t} M_{\nu_{R}}^{-1} Y_{\nu_{D}}\left\langle H_{u}\right\rangle^{2}$ if its mass is nothing but the value expected by the symmetry. Therefore, if one of the enhancement factors $\eta_{S_{i}}$ and $\eta_{N_{R i}^{c}}$ is around one, all components of $M_{\nu}$ becomes the values expected by the symmetry, and so are all components of the diagonalizing matrix. In order to obtain three eigenvalues expected by the symmetry, three of the six enhancement factors must be around one. Then all predictions on the light 
neutrino sector become the same order as the predictions without the enhancement factors. Since the lightest neutrino mass has only its upper limit fixed by experiments, the prediction for it can be different from the predicted value without any enhancement factor. Therefore, it is sufficient that two RH neutrinos have their masses which are determined by the symmetry for consistency with the present constraints obtained by neutrino experiments.

It looks not to be fair that we consider these enhancement effects only for the $\mathrm{RH}$ neutrino masses, although the mass terms have much larger $\mathrm{U}(1)_{A}$ charges than the other terms like Yukawa terms. We should change the $\mathrm{U}(1)_{A}$ charge assignment in table 1 , when such enhancement effects are taken into account. This subject is beyond the scope of this paper. Here we should emphasize that even after changing these $\mathrm{U}(1)_{A}$ charges, the mass terms of $S_{1}, S_{2}$, and $N_{R 1}^{c}$ have still much larger U(1) $A$ charges, and therefore, some enhancements for their masses are expected.

The next important question is how large enhancement factor is needed for sufficient leptogenesis in this $E_{6}$ GUT model. In the next section, we try to answer this question.

\section{Leptogenesis in the $E_{6} \times \mathrm{U}(1)_{A}$ model}

In the thermal leptogenesis scenario, thermally produced RH neutrinos go out of equilibrium as temperature decreases to their mass scale, and their $\mathrm{CP}$ asymmetric decays produce lepton asymmetry [33]. The lepton asymmetry is converted to the baryon asymmetry via the nonperturbative $B+L$ violating sphaleron processes [40].

In this section, we calculate the lepton number in the $E_{6} \times \mathrm{U}(1)_{A}$ model with the Dirac neutrino Yukawa couplings $Y_{\alpha i}(\alpha=1,2, \cdots, 6, i=1,2,3)$ which are determined by the symmetry as in table 2 and the masses $M_{\alpha}$ for the mass eigenstate of the RH neutrinos $N_{\alpha}$. Some of six $M_{\alpha}$ have enhancement factors $\eta_{\alpha}$ larger than 1 . What we would like to know by this calculation is how large enhancement factors are required to obtain sufficiently large lepton number. In the calculation, it is important to include supersymmetric contributions and the effects of lepton flavor in the final state of the decay process simultaneously. To show this statement, we calculate the lepton asymmetry in each case:

- non-SUSY + non flavor

- non-SUSY + flavor

- SUSY + non flavor

- SUSY + flavor

The result is shown in figure 1 . In a realistic situation of the $E_{6} \times \mathrm{U}(1)_{A}$ GUT model, i.e., in the case of SUSY+flavor, the sufficient lepton number can be obtained if the enhancement factor for the $N_{1}$ mass is around 16 . This means that $M_{1} \sim 9 \times 10^{8} \mathrm{GeV}$.

It is known that supersymmetric contribution is important when the decay parameter $K \equiv \Gamma_{N_{1}}(T=0) / H\left(T=M_{1}\right)$ is smaller than 1 because supersymmetric calculation makes $K$ larger effectively. On the other hand, the lepton flavor effects are important when $K$ is larger than 1 because $K$ for the muon and the electron become smaller than $K$ for the 


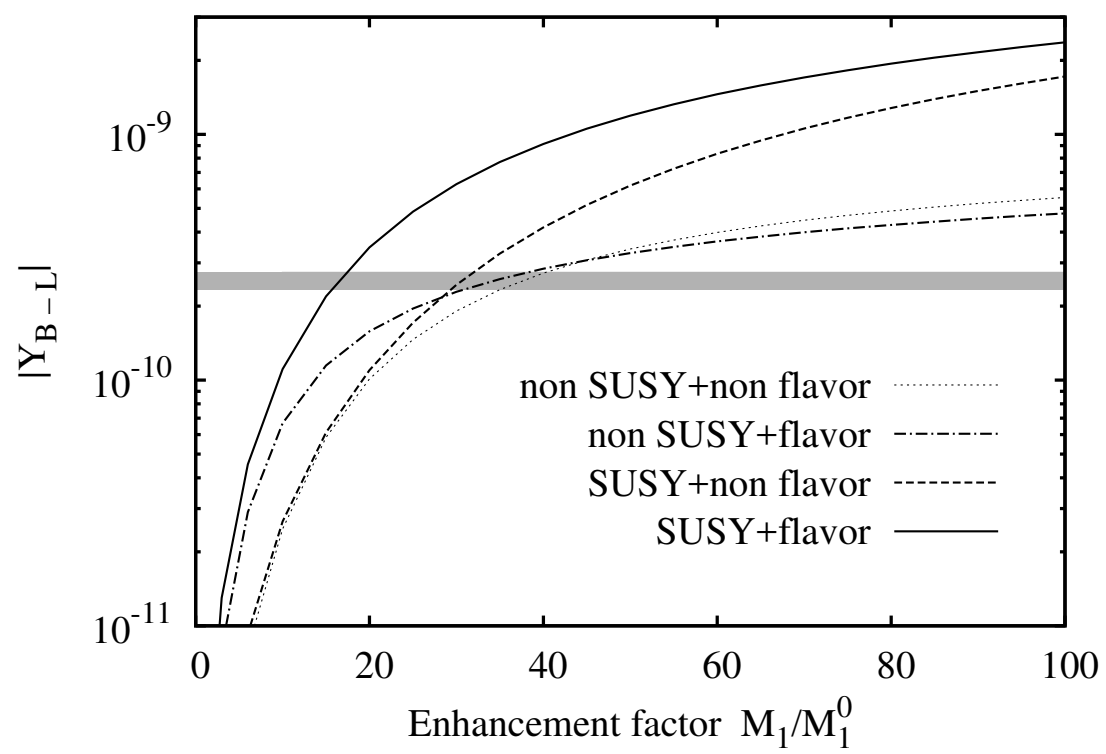

Figure 1. Enhancement factor dependence of $\left|Y_{B-L}\right|$ in each case. Horizontal band corresponds to the observed baryon asymmetry in SUSY cases. $M_{1}^{0}$ is the "bare" Majorana mass in the absence of the $\mathrm{U}(1)_{A}$ interactions. We take the simplified CP asymmetry $\epsilon_{1}^{\mathrm{SM}}(4.4), \epsilon_{1 i}^{\mathrm{SM}}(4.7), \epsilon_{1}^{\mathrm{SUSY}}=2 \times \epsilon_{1}^{\mathrm{SM}}$, and $\epsilon_{1 i}^{\mathrm{SUSY}}=2 \times \epsilon_{1 i}^{\mathrm{SM}}$ with the assumption $\Im\left[\left(Y^{\dagger} Y\right)_{61}\right]=\Re\left[\left(Y^{\dagger} Y\right)_{61}\right]$ in each case, respectively.

tau. Here $T$ is temperature of the universe. Our calculation shows that it is important to inculude both contributions when $K$ is larger than 1 . This is because supersymmetric contribution is important for smaller $K$ of the electron and the muon.

\section{1 non-SUSY + non-flavor}

In this subsection, we evaluate the lepton asymmetry in the non-SUSY+non-flavor case in $E_{6} \times \mathrm{U}(1)_{A}$ models after brief review.

In the model, since RH neutrinos are hierarchical in mass, the lepton asymmetry is generated by the $\mathrm{CP}$ asymmetric reactions of the lightest $\mathrm{RH}$ neutrino $N_{1}$. In the followings, we assume that the lightest RH neutrino is $N_{1}$ while it has the largest enhancement factor. The lepton asymmetry is evaluated by a coupled set of evolution equations of the lightest RH neutrino $N_{1}$ and the lepton asymmetry $L$ :

$$
\begin{aligned}
& \frac{d Y_{N_{1}}}{d z}=-\frac{z}{s H(z=1)}\left(\frac{Y_{N_{1}}}{Y_{N_{1}}^{e q}}-1\right)\left[\gamma_{D}+2 \gamma_{S s}+4 \gamma_{S t}\right], \\
& \frac{d Y_{L}}{d z}=-\frac{z}{s H(z=1)}\left\{\left[\frac{1}{2} \frac{Y_{L}}{Y_{l}^{e q}}+\epsilon_{N_{1}}^{\mathrm{SM}}\left(1-\frac{Y_{N_{1}}}{Y_{N_{1}}^{e q}}\right)\right] \gamma_{D}+\frac{Y_{L}}{Y_{l}^{e q}}\left[2 \gamma_{S t}+\frac{Y_{N_{1}}}{Y_{N_{1}}^{e q}} \gamma_{S s}\right]\right\} .
\end{aligned}
$$

Here $s$ is the entropy density, and $H$ is the Hubble parameter. We use a dimensionless variable $z \equiv M_{1} / T . \quad Y_{X}$ and $Y_{X}^{e q}$ are yield value and its equilibrium one of a species $X$, respectively, which are the number density normalized to the entropy density. $\gamma_{D}=$ $\gamma_{D}\left(N_{1} \leftrightarrow l H\right), \gamma_{S s}=\gamma_{S s}\left(N_{1} l \leftrightarrow Q_{3} T_{R}^{c}\right)$, and $\gamma_{S t}=\gamma_{S t}\left(N_{1} Q_{3} \leftrightarrow l T_{R}\right)$ are thermal averaged decay rate (inverse decay rate), s-channel, and t-channel scattering rate, respectively [41]. 


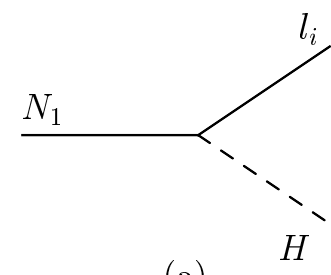

(a)

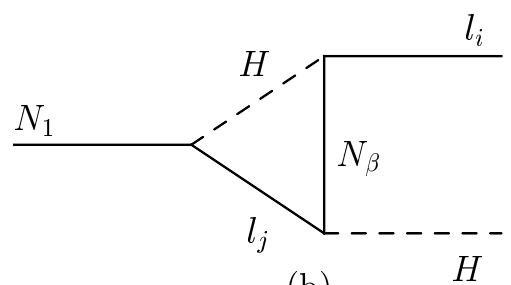

(b)

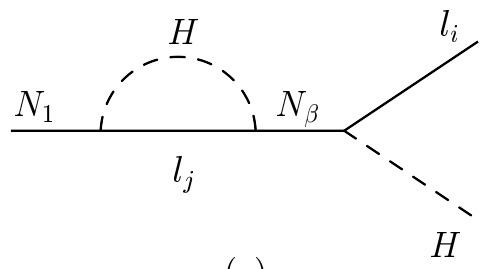

(c)

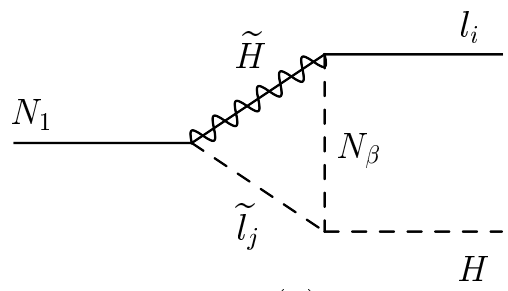

(d)

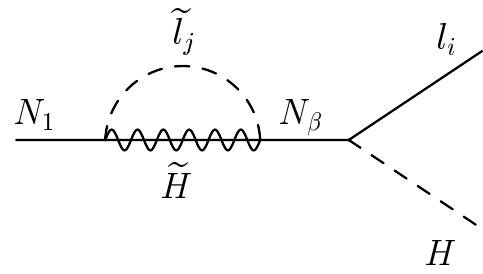

(e)

Figure 2. Tree and one-loop diagrams contributing to the CP asymmetric decay of lightst $\mathrm{RH}$ neutrino. In the model, five $\mathrm{RH}$ neutrinos $N_{\beta}(\beta=2, \ldots, 6)$ contribute to the $\mathrm{CP}$ asymmetry.

Here $l, Q_{3}$, and $T_{R}$ denote $\mathrm{SU}(2)_{L}$ doublet lepton, third generation doublet quark, and singlet top quark, respectively. The $C P$ asymmetry $\epsilon_{N_{1}}^{\mathrm{SM}}$ is defined as $\epsilon_{N_{1}}^{\mathrm{SM}}=\left[\Gamma\left(N_{1} \rightarrow\right.\right.$ $\left.l H)-\Gamma\left(N_{1} \rightarrow \bar{l} H^{\dagger}\right)\right] /\left[\Gamma\left(N_{1} \rightarrow l H\right)+\Gamma\left(N_{1} \rightarrow \bar{l} H^{\dagger}\right)\right]$. The first non-zero contribution to $\epsilon_{N_{1}}^{\mathrm{SM}}$ comes from interference between tree-level amplitude with the one-loop contributions (upper three diagrams in figure 2), and it is calculated in a hierarchical limit in RH neutrino masses as $\epsilon_{N_{1}}^{\mathrm{SM}}=-(3 / 16 \pi) \sum_{\beta \neq 1}^{6}\left(\Im\left[\left(Y^{\dagger} Y\right)_{\beta 1}^{2}\right] /\left[Y^{\dagger} Y\right]_{11}\right)\left(M_{1} / M_{\beta}\right)$ [42]. Note that, the $E_{6}$ GUT model has six RH neutrinos, and therefore, $\beta=2,3, \cdots, 6$.

Key ingredients for the lepton asymmetry generation are the CP asymmetry $\epsilon_{N_{1}}^{\mathrm{SM}}$ and the decay parameter $K \equiv \Gamma_{N_{1}}(T=0) / H\left(T=M_{1}\right)$ [43] which parametrizes the departure from the thermal equilibrium of $\mathrm{RH}$ neutrinos at $T=M_{1} . K$ is important because it is related with $\gamma_{D}$ and the factor $\left(1-Y_{N_{1}} / Y_{N_{1}}^{\text {eq }}\right)$ in eq. (4.2). The lepton asymmetry $Y_{L}$ is essentially determined by the above two parameters as

$$
Y_{L} \sim \epsilon_{N_{1}}^{\mathrm{SM}} C(K)
$$

The behavior of the function $C(K)$ is as follows. When $K>1, C(K)$ becomes a decreasing function of $K . K>1$ means that the $\mathrm{RH}$ neutrinos are still in the thermal equilibrium at $T=M_{1}$, and therefore, the number density of $N_{1}$ decreases rapidly when $T<M_{1}$. This reduces the produced lepton asymmetry. When $K<1, C(K)$ becomes a increasing function of $K . K<1$ means that the $\mathrm{RH}$ neutrinos do not reach the thermal equilibrium at $T=M_{1}$, and therefore, the number of thermally produced RH neutrinos becomes smaller for smaller $K$. This reduces the produced lepton asymmetry. Around $K \sim 1$, the function $C(K)$ becomes maximal. Sufficient lepton asymmetry can be obtained when $K \sim 1$ and $\epsilon_{N_{1}}^{\mathrm{SM}} \sim 10^{-7}$. 
Let us calculate the above two important parameters in the $E_{6} \times \mathrm{U}(1)_{A}$ GUT. First, we estimate $\epsilon_{N_{1}}^{\mathrm{SM}}$ and $K^{\mathrm{SM}}$ without any enhancement factor $\eta_{\alpha}$ as

$$
\begin{aligned}
\epsilon_{N_{1}}^{\mathrm{SM}} & =2\left(-\frac{3}{16 \pi} \frac{\Im\left[\left(Y^{\dagger} Y\right)_{61}^{2}\right]}{\left[Y^{\dagger} Y\right]_{11}} \frac{M_{1}}{M_{6}}\right) \sim-8.85 \times 10^{-9}\left(\frac{M_{1}}{5.7 \times 10^{7} \mathrm{GeV}}\right), \\
K^{\mathrm{SM}} & =\frac{\left[Y^{\dagger} Y\right]_{11} M_{1} / 8 \pi}{1.66\left(g_{*}^{\mathrm{SM}}\right)^{1 / 2} M_{1}^{2} / M_{\mathrm{pl}}} \simeq 37\left(\frac{5.7 \times 10^{7} \mathrm{GeV}}{M_{1}}\right),
\end{aligned}
$$

where $M_{\mathrm{pl}}$ is the Planck scale and $g_{*}^{\mathrm{SM}}$ is the effective relativistic degrees of freedom, which is obtained as $g_{*}^{\mathrm{SM}}=106.75$ with the SM particle contents. For the estimation of $\epsilon_{N_{1}}^{\mathrm{SM}}$, we have adopted two assumptions. The first assumption is that $\Im\left[\left(Y^{\dagger} Y\right)_{\beta 1}^{2}\right]$ can be estimated by $\Re\left[\left(Y^{\dagger} Y\right)_{\beta 1}^{2}\right]$. This assumption is reasonable because we regard all Yukawa couplings as complex numbers. The second assumption is on the factor 2 in front of the parenthesis in eq. (4.4). An important observation is that $\left(Y^{\dagger} Y\right)_{\beta 1}^{2}\left[Y^{\dagger} Y\right]_{11}^{-1} \frac{M_{1}}{M_{\beta}} \sim \lambda^{11}$ is not dependent on $\beta$. Therefore, we can expect an enhancement factor after summation of the index $\beta$, and we assume that the enhancement factor is two through all calculations in this paper.

It is obvious that the lepton asymmetry with these parameters are too small to explain the observation. $K^{\mathrm{SM}}$ is too large and $\epsilon_{S_{1}}^{\mathrm{SM}}$ is too small. However, as discussed in the previous section, the lightest $\mathrm{RH}$ neutrino mass can be expected to have an enhancement factor which can be much larger than one. Interestingly, when the lightest $\mathrm{RH}$ neutrino mass $M_{1}$ becomes larger, the produced lepton asymmetry becomes larger because the CP asymmetry $\epsilon_{N_{1}}^{\mathrm{SM}}$ becomes larger and the decay parameter $K^{\mathrm{SM}}$ becomes smaller as seen in eqs. (4.4) and (4.5). For example, if we take the enhancement factor is around 37, the sufficient lepton asymmetry can be expected because $K^{\mathrm{SM}} \sim 1$ and $\epsilon_{N_{1}}^{\mathrm{SM}} \sim 3 \times 10^{-7}$.

Figure 3 shows the evolutions of the lepton asymmetry $\left|Y_{B-L}\right|$, yield value of the RH neutrino $Y_{N_{1}}$, and its equilibrium one $Y_{N_{1}}^{e q}$ for $M_{1}=1 \times M_{1}^{0}$ (top panel) and $M_{1}=100 \times M_{1}^{0}$ (bottom panel). Here $M_{1}^{0}$ represents the "bare" Majorana mass, that is the physical mass of the lightest $\mathrm{RH}$ neutrino without any enhancement factor. The lepton asymmetry for $M_{1}=1 \times M_{1}^{0},\left|Y_{L}\right| \simeq 10^{-13}$, is too small to account for the observed baryon asymmetry. In non-SUSY cases, the required lepton asymmetry is $2.285 \times 10^{-10} \leq Y_{B-L} \leq 2.685 \times$ $10^{-10}$ with the conversion rate of the lepton asymmetry to the baryon asymmetry $Y_{B}=$ $(28 / 79) Y_{B-L}[44]$ and the observed baryon number $8.097 \times 10^{-11} \leq Y_{B} \leq 9.518 \times 10^{-11}$ [45]. For $M_{1}=100 \times M_{1}^{0}$, the lepton asymmetry is drastically enhanced. The enhancement of the lightest RH neutrino mass makes the CP asymmetry larger [see eq. (4.4)] and reduces the $K^{\mathrm{SM}}$ factor. Indeed, in the bottom panel in figure 3 , we find the larger deviation from thermal equilibrium compared with the top panel. The combination of these effects leads the enhancement of lepton asymmetry.

Dotted line in figure 1 shows the $M_{1}$ dependence of the lepton asymmetry in the nonSUSY+non-flavor case. Since numerically $C(K) \propto K$ for $K \ll 1$, the lepton asymmetry becomes asymptotically a constant for the enhancement factor larger than 40 . In this case, the physical mass of the $\mathrm{RH}$ neutrino is required to be $M_{1}=(35-39) \times M_{1}^{0}$ to account for the observed baryon number. 

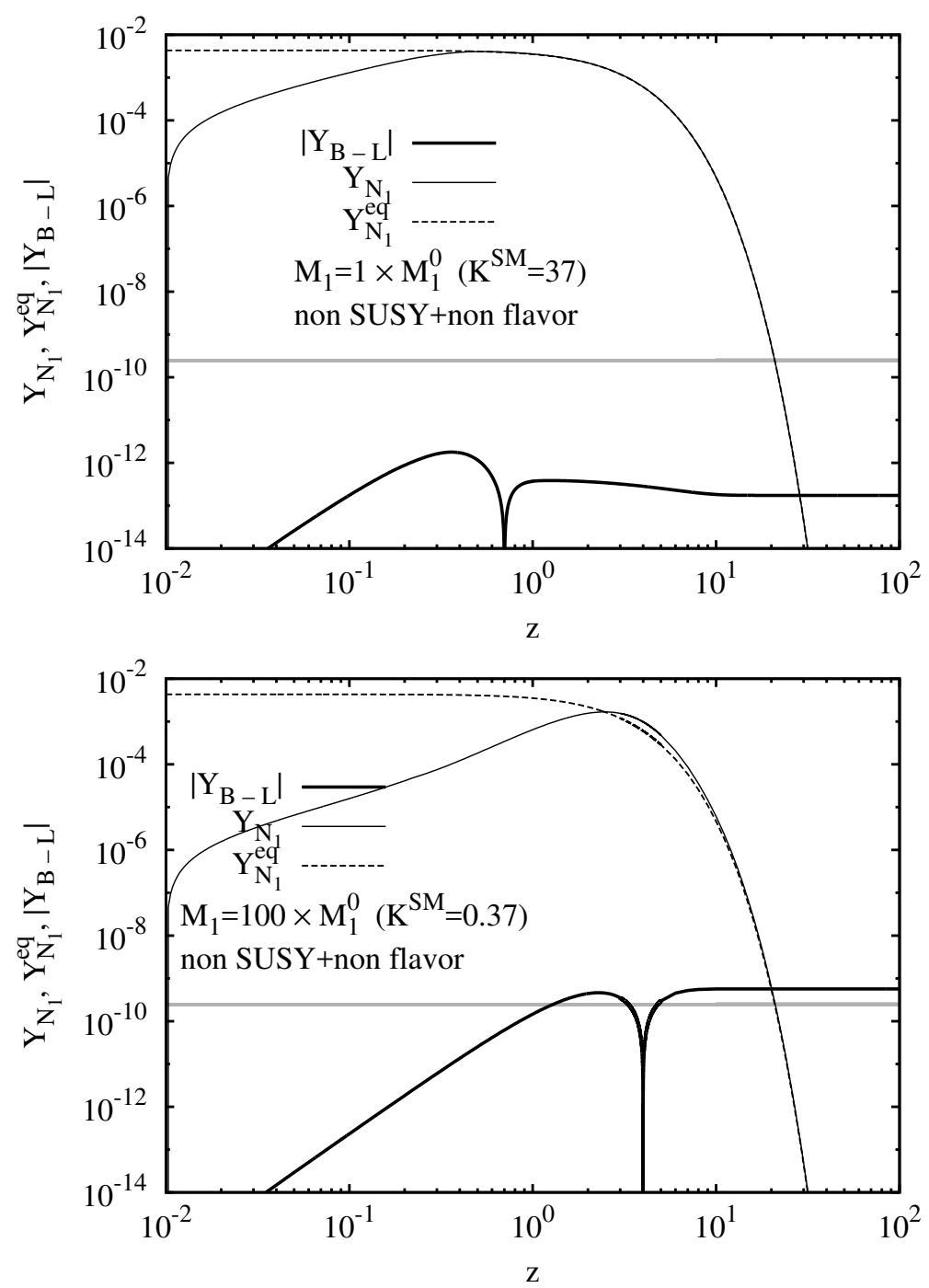

Figure 3. Evolutions of $\left|Y_{B-L}\right|, Y_{N_{1}}$, and $Y_{N_{1}}^{e q}$ for $M_{1}=1 \times M_{1}^{0}$ (top panel) and $M_{1}=100 \times M_{1}^{0}$ (bottom panel) in the non-SUSY+non-flavor case. Here $M_{1}^{0}$ is the "bare" Majorana mass in the absence of the $\mathrm{U}(1)_{A}$ interactions. Horizontal band corresponds to the observed baryon asymmetry. We take the simplified CP asymmetry (4.4) with the assumption $\Im\left[\left(Y^{\dagger} Y\right)_{61}\right]=\Re\left[\left(Y^{\dagger} Y\right)_{61}\right]$.

\subsection{Non-SUSY + flavor}

It is important for the evaluation of lepton asymmetry in the $E_{6} \times \mathrm{U}(1)_{A}$ model to separately involve each lepton flavor channel of the CP asymmetric decays. The reasons are as follows. The $E_{6}$ model possesses the features: (i) the evolution of lightest $\mathrm{RH}$ neutrino is in the strong washout regime, (ii) all of asymmetry productions of each lepton flavor by the CP asymmetric decay are sizable. These features can give rise to $\mathcal{O}(1)$ corrections to the final lepton asymmetry with respect to the case where the flavor effects are ignored [46, 47]. This is because that the evolutions of the lepton asymmetries of each lepton flavor are in the regime of washout with different magnitudes. In this section we briefly review the leptogenesis with the flavor effects. 
The total lepton asymmetry is given by the sum of the asymmetry of each lepton flavor, $Y_{B-L}=Y_{\Delta_{e}}+Y_{\Delta_{\mu}}+Y_{\Delta_{\tau}}$, where $\Delta_{i}=B / 3-L_{i}$. The subscript $i$ represents the lepton flavor. The evolutions of each asymmetry is described by the flavor dependent Boltzmann equations,

$$
\begin{aligned}
\frac{d Y_{\Delta_{i}}}{d z}= & -\frac{z}{s H(z=1)}\left\{\left(\frac{Y_{N_{1}}}{Y_{N_{1}}^{e q}}-1\right) \epsilon_{1 i}^{\mathrm{SM}} \gamma_{D}+K_{i}^{0} \sum_{j}\left[\frac{1}{2}\left(C_{i j}^{l}+C_{j}^{H}\right) \gamma_{D}\right.\right. \\
& \left.\left.+\left(\frac{Y_{N_{1}}}{Y_{N_{1}}^{e q}}-1\right)\left(C_{i j}^{l} \gamma_{S_{s}}+\frac{C_{j}^{H}}{2} \gamma_{S_{t}}\right)+\left(2 C_{i j}^{l}+C_{j}^{H}\right)\left(\gamma_{S_{t}}+\frac{\gamma_{S_{s}}}{2}\right)\right] \frac{Y_{\Delta_{i}}}{Y_{l}^{e q}}\right\} .
\end{aligned}
$$

The coefficient $K_{i}^{0}$ is the flavor projection, $K_{i}^{0}=Y_{1 i} Y_{1 i}^{*} /\left(Y Y^{\dagger}\right)_{11}$. The flavor dependent $\mathrm{CP}$ asymmetry is defined as $\epsilon_{1 i}^{\mathrm{SM}}=\left[\Gamma\left(N_{1} \rightarrow l_{i} H\right)-\Gamma\left(N_{1} \rightarrow \bar{l}_{i} H^{\dagger}\right)\right] /\left[\Gamma\left(N_{1} \rightarrow\right.\right.$ $\left.\left.l_{i} H\right)+\Gamma\left(N_{1} \rightarrow \bar{l}_{i} H^{\dagger}\right)\right]$, and is calculated in the hierarchical mass limit as $\epsilon_{1 i}^{\mathrm{SM}}=$ $-\left(1 / 8 \pi\left(Y Y^{\dagger}\right)_{11}\right) \sum_{\beta \neq 1}^{6} \Im\left\{Y_{\beta i} Y_{1 i}^{*}\left[(3 / 2)\left(M_{1} / M_{\beta}\right)\left(Y Y^{\dagger}\right)_{\beta 1}+\left(M_{1}^{2} / M_{\beta}^{2}\right)\left(Y Y^{\dagger}\right)_{1 \beta}\right]\right\}[42]$. We follow the considerations for deriving eq. (4.4), and obtain the simplified CP asymmetry in the non-SUSY+flavor case as

$$
\epsilon_{1 i}^{\mathrm{SM}}=2\left(\frac{-1}{8 \pi\left(Y Y^{\dagger}\right)_{11}} \Im\left\{Y_{6 i} Y_{1 i}^{*}\left[\frac{3}{2} \frac{M_{1}}{M_{6}}\left(Y Y^{\dagger}\right)_{61}+\frac{M_{1}^{2}}{M_{6}^{2}}\left(Y Y^{\dagger}\right)_{16}\right]\right\}\right)
$$

The coefficient $C^{l}\left(C^{H}\right)$ in eq. (4.6) is introduced as the conversion factor between the asymmetry normalized to the equilibrium number density for $l_{i}(H)$ and the yield value of each lepton number normalized to equilibrium lepton density as $\left(n_{l_{i}}-n_{\bar{l}_{i}}\right) / n_{l_{i}}^{\text {eq }}=$ $-\sum_{j} C_{i j}^{l}\left(Y_{\Delta_{j}} / Y_{l}^{\mathrm{eq}}\right)$, and $\left(n_{H}-n_{\bar{H}}\right) / n_{H}^{\mathrm{eq}}=-\sum_{j} C_{j}^{H}\left(Y_{\Delta_{j}} / Y_{l}^{\mathrm{eq}}\right)$. The entries are modelindependent, which are determined by constraints among the chemical potentials enforced by the equilibrium reactions in the temperature $T \sim M_{1}$ where the asymmetries are generated. The region of RH neutrino mass we consider is $1 \leq M_{1} / M_{1}^{0} \leq 100$, and the relevant temperature of the leptogenesis in the model is in $10^{5} \mathrm{GeV} \lesssim T \lesssim 10^{11} \mathrm{GeV}$. In this range, SM gauge interactions, third and second generation Yukawa interactions are in equilibrium, and the equilibrium conditions lead to the following $C^{l}$ and $C^{H}[47]$,

$$
C_{i j}^{l}=\frac{1}{2148}\left(\begin{array}{ccc}
906 & -120 & -120 \\
-75 & 688 & -28 \\
-75 & -28 & 688
\end{array}\right), \quad C^{H}=\frac{1}{358}\left(\begin{array}{lll}
37 & 52 & 52
\end{array}\right)
$$

Figure 4 shows the evolutions of total lepton asymmetry $\left|Y_{B-L}\right|$, and of the asymmetry of each lepton flavor $\left|Y_{\Delta_{i}}\right|(i=e, \mu, \tau)$ for $M_{1}=1 \times M_{1}^{0}$ (top panel) and for $M_{1}=100 \times M_{1}^{0}$ (bottom panel). For the interpretation of the result, we need to see both the magnitude of the washout and the production efficiency of each lepton asymmetry. We rearrange the $K^{\mathrm{SM}}$ factor (4.5) to involve the flavor dependence as $K_{i}^{\mathrm{SM}}=K_{i}^{0} K^{\mathrm{SM}}$. Each $K_{i}^{\mathrm{SM}}$ is 
obtained as follows,

$$
\begin{aligned}
& K_{e}^{\mathrm{SM}}=\frac{\Gamma_{N_{1} \rightarrow l_{e} H}^{\mathrm{SM}}(T=0)}{H\left(T=M_{1}\right)} \simeq 1.4\left(\frac{5.7 \times 10^{7} \mathrm{GeV}}{M_{1}}\right), \\
& K_{\mu}^{\mathrm{SM}}=\frac{\Gamma_{N_{1} \rightarrow l_{\mu} H}^{\mathrm{SM}}(T=0)}{H\left(T=M_{1}\right)} \simeq 6.4\left(\frac{5.7 \times 10^{7} \mathrm{GeV}}{M_{1}}\right), \\
& K_{\tau}^{\mathrm{SM}}=\frac{\Gamma_{N_{1} \rightarrow l_{\tau} H}^{\mathrm{SM}}(T=0)}{H\left(T=M_{1}\right)} \simeq 29\left(\frac{5.7 \times 10^{7} \mathrm{GeV}}{M_{1}}\right) .
\end{aligned}
$$

The $K_{i}^{\mathrm{SM}}$ is a measure of magnitude of the washout of each lepton asymmetry, that is the same with the relation between the $K^{\mathrm{SM}}$ factor and the washout of total lepton asymmetry. On the other hand, the ratio of asymmetry productions of each lepton flavor by the CP asymmetric decay is equal to the ratio of each $\mathrm{CP}$ asymmetry, and is obtained as follows

$$
\begin{aligned}
& \frac{L_{e} \text { production }}{L_{\tau} \text { production }}=\frac{\Im\left[Y_{61} Y_{11}^{*}\right]}{\Im\left[Y_{63} Y_{13}^{*}\right]}=0.048, \\
& \frac{L_{\mu} \text { production }}{L_{\tau} \text { production }}=\frac{\Im\left[Y_{62} Y_{12}^{*}\right]}{\Im\left[Y_{63} Y_{13}^{*}\right]}=0.220 .
\end{aligned}
$$

Here we assumed $\Im\left[Y_{6 i} Y_{1 i}^{*}\right]=\Re\left[Y_{6 i} Y_{1 i}^{*}\right]$. For $M_{1}=1 \times M_{1}^{0}, K_{e}$ and $K_{\mu}$ are $\mathcal{O}(1)$, but $K_{\tau} \simeq 29$. Each lepton asymmetry is generated by the CP asymmetric decays, and then a large part of the tau's is washed out by the inverse decays and so on, while the electron's and the muon's soon decouple from the equilibrium and survive. Thus, nonetheless the production efficiencies of the electron and the muon number are lower than the tau's, they yields a large part of the lepton asymmetry. On the other hand, for $M_{1}=100 \times M_{1}^{0}$, $K_{\tau} \simeq \mathcal{O}\left(10^{-1}\right)$, and $K_{e}, K_{\mu} \simeq \mathcal{O}\left(10^{-2}\right)$. These $K_{i}$ factors indicate that each lepton number generated by the $\mathrm{CP}$ asymmetric decay survives without washout. Thus a large part of lepton asymmetry is governed by the tau's.

The chain line in figure 1 shows the $M_{1}$ dependence of the total lepton asymmetry in the non-SUSY+flavor case. In the non-SUSY+flavor case, the physical mass of the RH neutrino is required to be $30 \leq M_{1} / M_{1}^{0} \leq 37$ to account for the observed baryon number. For $M_{1} / M_{1}^{0} \lesssim 40$, in the non-SUSY+non-flavor case, the evolution of the lepton asymmetry is in the strong washout regime. While, in the non-SUSY+flavor case, the muon and the electron asymmetries are in the weak washout regime, and yield sizable contribution to total lepton asymmetry. The lepton asymmetry with the flavor effects is therefore larger than that in the case where the effects are ignored. On the other hand, for $M_{1} / M_{1}^{0} \gtrsim 40$, the evolutions of the asymmetries of all lepton flavor are in the weak washout regime, and hence total lepton asymmetry is determined by only the asymmetry production by the CP asymmetric decay. Due to the additional washout contributions (spectator process), in the parameter region, the final lepton asymmetry can be smaller than that the case without the flavor effects $[46,47]$. 

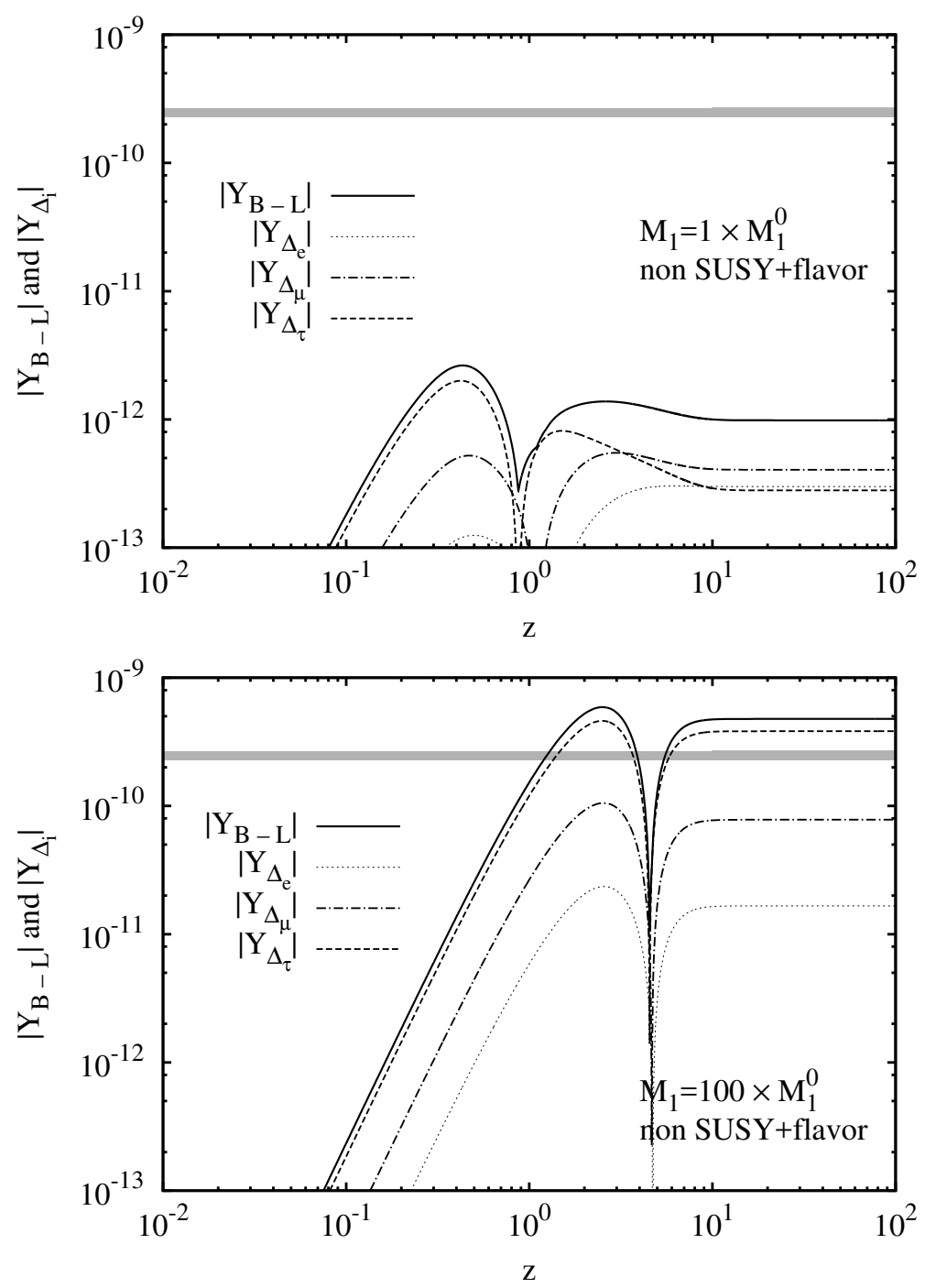

Figure 4. Same as figure 3, but in the non-SUSY+flavor case. The evolutions of electron number $L_{e}$, muon number $L_{\mu}$, and tau number $L_{\tau}$ are also plotted. Since the evolution of RH neutrino is same as the non-SUSY+non-flavor case, we cut the part.

\subsection{SUSY + non-flavor}

The SUSY extension of the leptogenesis gives an enhancement for the lepton asymmetry which is roughly estimated as

$$
\frac{Y_{B-L}^{\mathrm{SUSY}}}{Y_{B-L}^{\mathrm{SM}}} \sim \begin{cases}\sqrt{2} & \text { (strong washout) } \\ 2 \sqrt{2} & \text { (weak washout })\end{cases}
$$

in ref. [48]. In this section, we briefly review the corrections to interpret the numerical results in the context of the $E_{6} \times \mathrm{U}(1)_{A}$ model.

We have two important points which increase the lepton asymmetry. The additional decay channels correct the definition of the CP asymmetry as $\epsilon_{N_{1}}^{\mathrm{SUSY}}=\left[\Gamma\left(N_{1} \rightarrow\right.\right.$ $\left.l H)-\Gamma\left(N_{1} \rightarrow \bar{l} H^{\dagger}\right)+\Gamma\left(N_{1} \rightarrow \tilde{l} \tilde{H}\right)-\Gamma\left(N_{1} \rightarrow \tilde{l}^{*} \overline{\tilde{H}}\right)\right] / \Gamma_{N_{1}}^{\text {SUSY }} . \tilde{l}$ and $\tilde{H}$ represent SUSY 
partners of the $\mathrm{SU}(2)_{L}$ lepton and the Higgs doublet, respectively. Similarly, for the RH sneutrino, $\epsilon_{\tilde{N}_{1}}^{\mathrm{SUSY}}=\left[\Gamma\left(\tilde{N}_{1} \rightarrow \tilde{l} H\right)-\Gamma\left(\tilde{N}_{1} \rightarrow \tilde{l}^{*} H^{\dagger}\right)+\Gamma\left(\tilde{N}_{1} \rightarrow l \tilde{H}\right)-\Gamma\left(\tilde{N}_{1} \rightarrow \bar{l} \overline{\tilde{H}}\right)\right] / \Gamma_{\tilde{N}_{1}}^{\mathrm{SUUS}}$. Here $\Gamma_{\tilde{N}_{1}}^{\mathrm{SUSY}}$ is the total width of the RH sneutrino. The CP asymmetry receives the contributions of not only the RH neutrinos also of its scalar partner, and is obtained as $\epsilon_{N_{1}}^{\mathrm{SUSY}}=-(3 / 8 \pi) \sum_{\beta \neq 1}^{6}\left(\Im\left[\left(Y^{\dagger} Y\right)_{\beta 1}^{2}\right] /\left(Y^{\dagger} Y\right)_{11}\right)\left(M_{1} / M_{\beta}\right)$ in the hierarchical limit of RH neutrino masses [42]. The $\mathrm{CP}$ asymmetry of the $\mathrm{RH}$ sneutrino is equal to that of the $\mathrm{RH}$ neutrino in the hierarchical mass limit. Repeating the consideration for deriving eq. (4.4), we obtain the simplified CP asymmetries in the SUSY+non-flavor case as follows,

$$
\epsilon_{N_{1}}^{\mathrm{SUSY}}=\epsilon_{\tilde{N}_{1}}^{\mathrm{SUSY}}=2\left(-\frac{3}{8 \pi} \frac{\Im\left[\left(Y^{\dagger} Y\right)_{61}^{2}\right]}{\left(Y^{\dagger} Y\right)_{11}} \frac{M_{1}}{M_{6}}\right)=2 \times \epsilon_{N_{1}}^{\mathrm{SM}} .
$$

These effects make the lepton asymmetry four times larger.

The effective relativistic degrees of freedom $g_{*}^{\mathrm{SUSY}}=228.75$ is about twice of $g_{*}^{\mathrm{SM}}$, which reduces the lepton asymmetry to entropy ratio by $1 / 2$.

The most important one is the correction of $K$ factor. In the context of SUSY, the total width is obtained as $\Gamma_{N_{1}}^{\mathrm{SUSY}}=\Gamma\left(N_{1} \rightarrow l H\right)+\Gamma\left(N_{1} \rightarrow \bar{l} H^{\dagger}\right)+\Gamma\left(N_{1} \rightarrow \tilde{l} \tilde{H}\right)+\Gamma\left(N_{1} \rightarrow\right.$ $\left.\tilde{l}^{*} \overline{\tilde{H}}\right)=\left[Y^{\dagger} Y\right]_{11} M_{1} / 4 \pi=2 \times \Gamma_{N_{1}}^{\mathrm{SM}}$. The $K$ factor is calculated as follows,

$$
K^{\mathrm{SUSY}}=\frac{\Gamma_{N_{1}}^{\mathrm{SUSY}}(T=0)}{H\left(T=M_{1}\right)}=\frac{\left[Y^{\dagger} Y\right]_{11} M_{1} / 4 \pi}{1.66\left(g_{*}^{\mathrm{SUSY}}\right)^{1 / 2} M_{1}^{2} / M_{\mathrm{pl}}} \simeq 51\left(\frac{5.7 \times 10^{7} \mathrm{GeV}}{M_{1}}\right) .
$$

Roughly, the factor $K^{\mathrm{SUSY}}$ is $\sqrt{2}$ times larger than that in the SM. This effect reduces the lepton asymmetry in strong washout regime and enhances it in weak washout regime.

Finally we note the conversion rate from the lepton asymmetry to the baryon asymmetry. In the context of SUSY, the additional equilibrium reactions at the temperature $T \simeq M_{1}$ alter the constraints among the chemical potentials. The alteration leads the conversion rate as $Y_{B}=(8 / 23) Y_{B-L}$ [49]. Consequently the required lepton asymmetry in SUSY cases is $2.328 \times 10^{-10} \lesssim\left|Y_{B-L}\right| \lesssim 2.736 \times 10^{-10}$.

With all these effects, the result in eq. (4.13) is obtained.

Dashed line in figure 1 shows the $M_{1}$ dependence of the lepton asymmetry in the SUSY+non-flavor case. The lepton asymmetry is given by a sum of partial asymmetries from the $\mathrm{CP}$ asymmetric decays of $\mathrm{RH}$ neutrino and its scalar partner. It is evaluated by a coupled set of evolution equations of the RH neutrino, its scalar partner, and the partial asymmetries [41]. Due to too strong washout, for $M_{1} \lesssim 30 \times M_{1}^{0}$, nonetheless the additional CP asymmetric decays, the lepton asymmetry is close to that in the nonSUSY+non-flavor case. While, for $M_{1} \gtrsim 30 \times M_{1}^{0}$, because of both $K \simeq \mathcal{O}(1)$ and the additional CP asymmetric decays, larger lepton asymmetry is generated than those in the cases of non-SUSY+non-flavor and non-SUSY+flavor.

\subsection{SUSY + flavor}

We are now in a position to discuss the lepton asymmetry in the SUSY+flavor case, which involves the SUSY particles contributions with the flavor effects [see section 4.2]. This is the realistic situation in the $E_{6} \times \mathrm{U}(1)_{A}$ model. Interestingly, even in the strong washout 
regime which is defined by $K^{\mathrm{SM}}>1$, the effect of SUSY becomes sizable if the flavor effects are included.

The sum of total lepton and slepton asymmetries, $Y_{L}^{(f)}$ and $Y_{L}^{(s)}$, converts to the baryon asymmetry, and which are given by the sum of the asymmetry of each lepton and slepton flavor: $Y_{B-L}=Y_{L}^{(f)}+Y_{L}^{(s)}=\left(Y_{\Delta_{e}}+Y_{\Delta_{\mu}}+Y_{\Delta_{\tau}}\right)+\left(Y_{\tilde{\Delta}_{e}}+Y_{\tilde{\Delta}_{\mu}}+Y_{\tilde{\Delta}_{\tau}}\right)$. We take the SUSY spectrum to be $\mathcal{O}(1 \mathrm{TeV})$ in the $E_{6} \times \mathrm{U}(1)_{A}$ model. Then, throughout the temperature region we consider, the equality of chemical potentials of a SM particle and its superpartner, which is refferd as superequilibration [50], is maintained in the presence of equilibrium supergauge (or Yukawa) reactions. In the superequilibrium regime, since the equality of asymmetries of each lepton and its scalar partner is also maintained, $Y_{B-L}=$ $2 \times\left(Y_{\Delta_{e}}+Y_{\Delta_{\mu}}+Y_{\Delta_{\tau}}\right)$. Thus the baryon asymmetry in the case is evaluated by a coupled set of evolution equations of the RH neutrino, its scalar partner, and asymmetry of each lepton flavor. The flavor dependent Boltzmann equations are shown in ref. [51], and relevant cross sections are given in ref. [41].

Figure 5 shows the $M_{1}$ dependence of the lepton asymmetry $\left|Y_{B-L}\right|$ and the partial asymmetry of each lepton flavor $\left|Y_{\Delta_{i}}\right|$. From the figure 5, the enhancement factor $16 \leq M_{1} / M_{1}^{0} \leq 17$ can yield the observed baryon number. The $M_{1}$ dependence of the asymmetries are described by the washout effects and production efficiencies of the asymmetries, that is basically the same as in the non-SUSY+flavor case. The magnitude of washout of each lepton flavor is parametrized by $K_{i}^{\mathrm{SUSY}}$, which is SUSY extension of $K_{i}^{\mathrm{SM}}$ [eqs. (4.9)-(4.11)]:

$$
\begin{aligned}
& K_{e}^{\mathrm{SUSY}}=\frac{\Gamma_{N_{1} \rightarrow l_{e} H}^{\mathrm{SUSY}}(T=0)}{H\left(T=M_{1}\right)} \simeq 1.9\left(\frac{5.7 \times 10^{7} \mathrm{GeV}}{M_{1}}\right), \\
& K_{\mu}^{\mathrm{SUSY}}=\frac{\Gamma_{N_{1} \rightarrow l_{\mu} H}^{\mathrm{SUSY}}(T=0)}{H\left(T=M_{1}\right)} \simeq 8.8\left(\frac{5.7 \times 10^{7} \mathrm{GeV}}{M_{1}}\right), \\
& K_{\tau}^{\mathrm{SUSY}}=\frac{\Gamma_{N_{1} \rightarrow l_{\tau} H}^{\mathrm{SUSY}}(T=0)}{H\left(T=M_{1}\right)} \simeq 40\left(\frac{5.7 \times 10^{7} \mathrm{GeV}}{M_{1}}\right) .
\end{aligned}
$$

As in the non-flavor case, these $K$ factors become $\sqrt{2}$ times larger than in the non-SUSY case. These corrections make the washout of each asymmetry stronger compared with nonSUSY case and the SUSY effects become weak especially in the strong washout regime. However, even if $K^{\mathrm{SM}}>1$, some of the $K_{i}^{\mathrm{SUSY}}$ can be $\sim \mathcal{O}(1)$, and therefore, the washout effect for the flavor $i$ is negligible. Then the supersymmetric contribution become sizable. Consequently, the lepton asymmetry generation is sufficiently boosted compared with the case of the non-flavor especially when $K^{\mathrm{SM}}>1$.

Figure 6 shows the evolutions of total lepton asymmetry $\left|Y_{B-L}\right|$ and partial asymmetries of each lepton flavor $\left|Y_{\Delta_{i}}\right|$ for $M_{1} / M_{1}^{0}=16$. To understand the importance of the flavor effects in SUSY calculation, we plot figure 7 in which the ratios of SUSY lepton asymmetry to non-SUSY lepton asymmetry are plotted. First of all, the figure shows that SUSY enhancement factor is larger in the weak washout regime than in the strong washout regime as explained in the previous subsection. Next, the figure shows that the SUSY enhancement factor with the flavor effect is larger than without the flavor effect. Especially, 


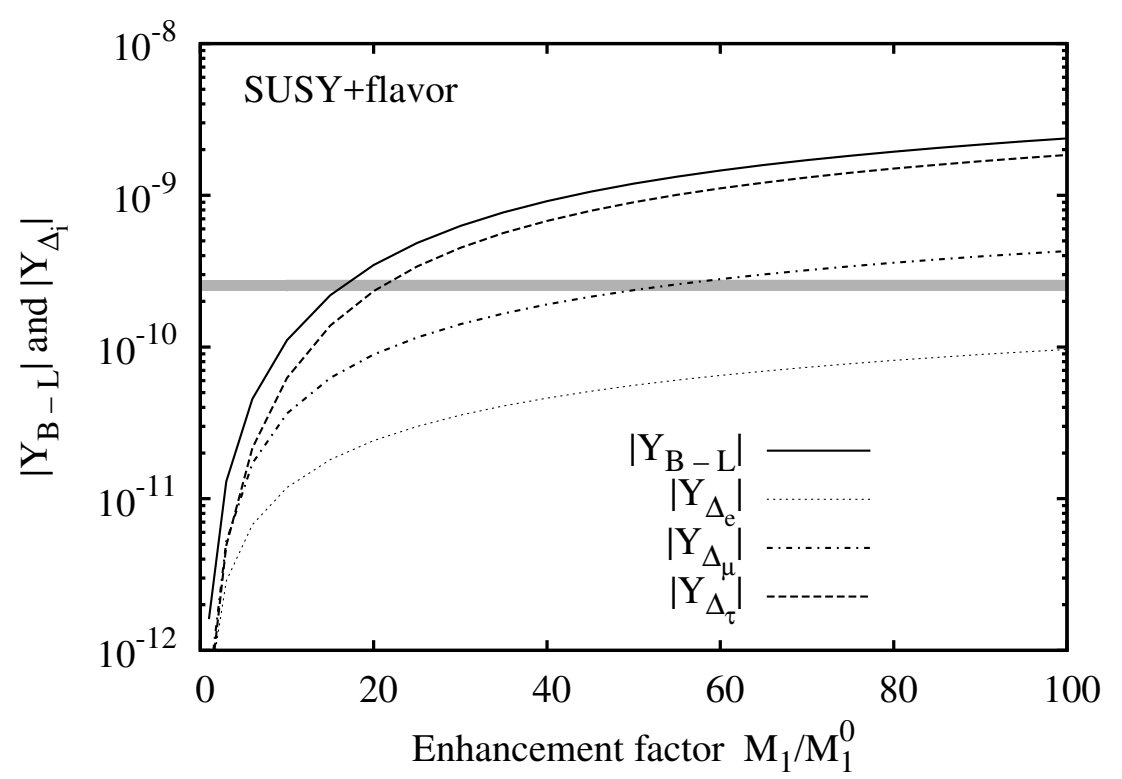

Figure 5. Enhancement factor dependence of $\left|Y_{B-L}\right|$ and $\left|Y_{\Delta_{i}}\right|$ in the SUSY+flavor case. Horizontal band corresponds to the observed baryon asymmetry. We take the simplified CP asymmetry $\epsilon_{1 i}^{\mathrm{SUSY}}=2 \times \epsilon_{1 i}^{\mathrm{SM}}$ with the assumption $\Im\left[\left(Y^{\dagger} Y\right)_{61}\right]=\Re\left[\left(Y^{\dagger} Y\right)_{61}\right]$, where $\epsilon_{1 i}^{\mathrm{SM}}$ is given by $(4.7)$.

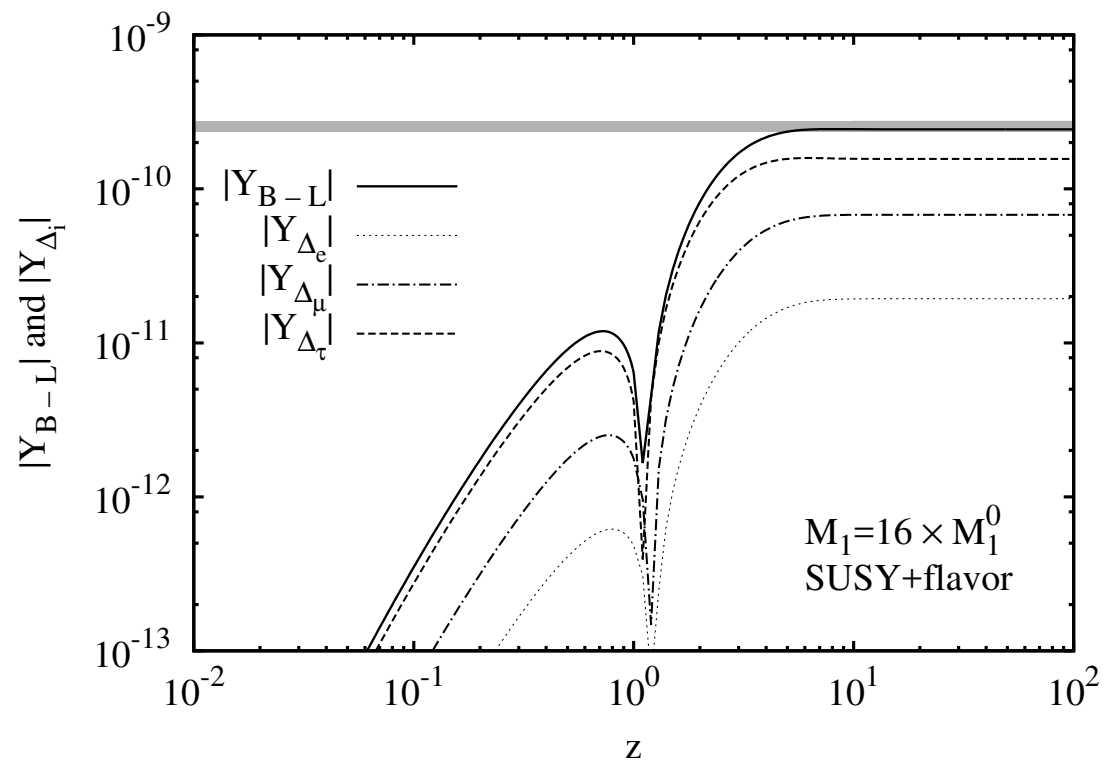

Figure 6. Evolutions of $\left|Y_{B-L}\right|$ and $\left|Y_{\Delta_{i}}\right|$ for $M_{1}=16 \times M_{1}^{0}$ in the SUSY+flavor case. Horizontal band corresponds to the observed baryon asymmetry. We take the simplified CP asymmetry $\epsilon_{1 i}^{\mathrm{SUSY}}=2 \times \epsilon_{1 i}^{\mathrm{SM}}$ with the assumption $\Im\left[\left(Y^{\dagger} Y\right)_{61}\right]=\Re\left[\left(Y^{\dagger} Y\right)_{61}\right]$, where $\epsilon_{1 i}^{\mathrm{SM}}$ is given by $(4.7)$.

it is important that even in the strong washout regime, the SUSY enhancement factor $\left|Y_{B-L}\right|_{\mathrm{SUSY}}^{\mathrm{f}} /\left|Y_{B-L}\right|_{\mathrm{SM}}^{\mathrm{f}}$ become sizable due to the enhancements of the muon and electron asymmetry, because $\left|Y_{B-L}\right|_{\mathrm{SM}}^{\mathrm{f}}$ has already been fairly larger than $\left|Y_{B-L}\right|_{\mathrm{SM}}^{\text {non-f }}$ in the strong washout regime. 


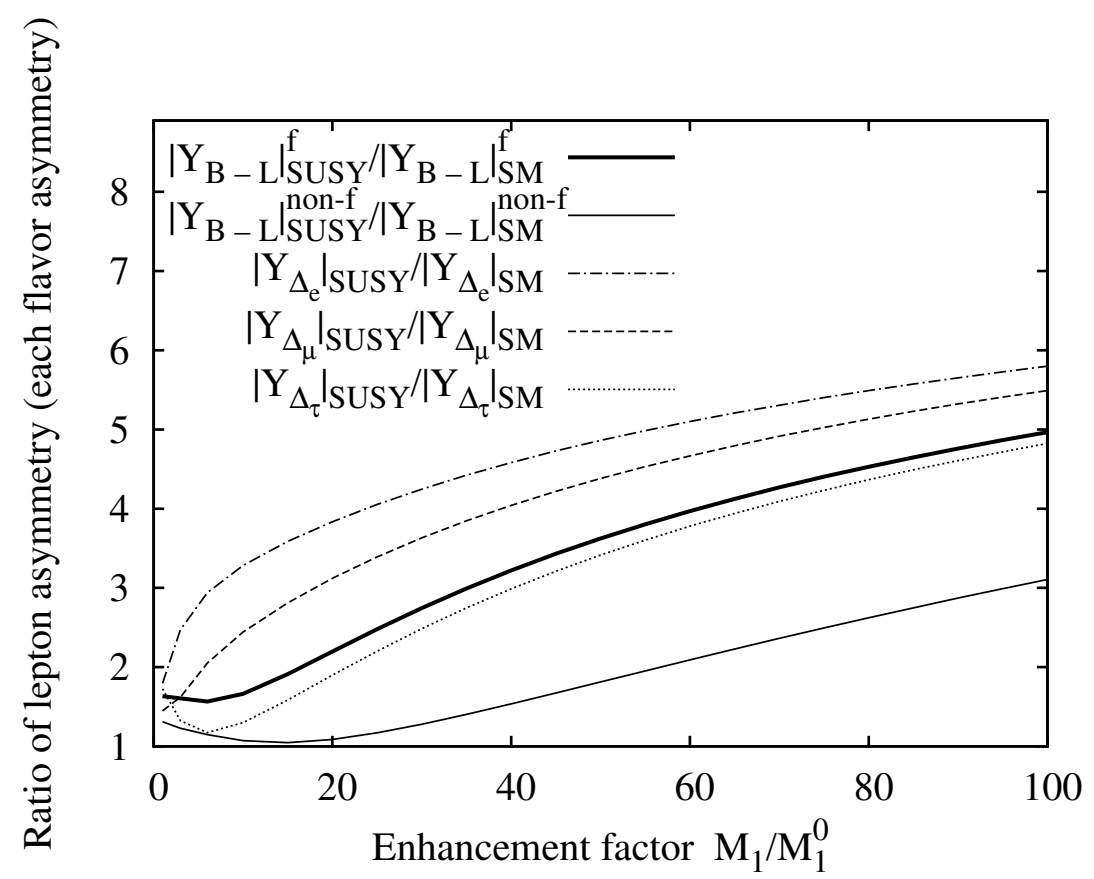

Figure 7. Enhancement factor dependence of the ratio of flavor contributions in each case, $\left(\left|Y_{\Delta_{i}}\right|\right)_{\mathrm{SUSY}} /\left(\left|Y_{\Delta_{i}}\right|\right)_{\mathrm{SM}},\left(\left|Y_{B-L}\right|\right)_{\mathrm{SUSY}}^{\mathrm{f}} /\left(\left|Y_{B-L}\right|\right)_{\mathrm{SM}}^{\mathrm{f}},\left(\left|Y_{B-L}\right|\right)_{\mathrm{SUSY}}^{\text {non-f }} /\left(\left|Y_{B-L}\right|\right)_{\mathrm{SM}}^{\text {non-f }}$. Here, for example, $\left|Y_{B-L}\right|_{\mathrm{SM}}^{\mathrm{f}}$ is the lepton asymmetry in the case non-SUSY+flavor, and $\left|Y_{B-L}\right|_{\mathrm{SUSY}}^{\text {non-f }}$ is the lepton asymmetry in the case SUSY+non-flavor, etc.

We could confirm the successful baryon asymmetry in the SUSY+flavor case, namely, in a realistic situation of the $E_{6} \times \mathrm{U}(1)_{A}$ GUT model. It is sufficient to take the lightest $\mathrm{RH}$ neutrino mass $M_{1} \sim 16 \times M_{1}^{0} \sim 9 \times 10^{8} \mathrm{GeV}$ for the observed baryon asymmetry. It is important for this calculation that all components of neutrino Yukawa matrix are determined by the symmety in the $E_{6} \times \mathrm{U}(1)_{A}$ GUT and we can integrate the flavor effects on the lepton asymmetry.

\section{Other baryogenesis}

Here we comment on other possibilities of baryogenesis in our scenario. Thermal leptogenesis in our scenario requires high reheating temperature, $T_{R} \gtrsim 10^{8} \mathrm{GeV}$. While, successful big-bang nucleosynthesis (BBN) leads to the constraint on gravitino abundance, which is translated into the upper bound on the reheating temperature [52-59]. It is called the gravitino problem. In this work we assume a way out from the contradiction, that is the heavy gravitino scenario, wherein the gravitino decays before the BBN and the bound on the reheating temperature is loosen [60-62]. Another possible way out is non-thermal leptogenesis scenarios [63-65].

The lightest $\mathrm{RH}$ neutrinos are non-thermally produced by inflaton decays, and generate the lepton asymmetry by the $\mathrm{CP}$ asymmetric decays (figure 2). Reheating temperature lower than the RH neutrino mass automatically leads to the out-of-equilibrium condition, and can make scenarios to be free from the gravitino problem. This is well motivated mechanism for the scenarios predicting relatively light gravitino. It is however difficult 
to quantitatively compute the amount of lepton asymmetry, because the generated lepton asymmetry strongly depends on the inflaton mass, the branching ratio for the mode $\phi \rightarrow$ $N_{1} N_{1}$ (here $\phi$ represents inflaton), and so on [66]. We should seriously discuss the lepton asymmetry via the inflaton decay with some specific inflation models after the reliable signal of light gravitino at collider experiments in future.

Affleck-Dine (AD) mechanism also gives rise to non-thermal generation of lepton (or baryon) asymmetry $[67,68]$. Some of slepton and squark fields condense with non-zero vacuum expectation values (VEVs) along the flat direction in field space during inflation, and store the lepton (or baryon) asymmetry through the quantum fluctuations of these fields. When inflation is over the asymmetries stored in the condensations are transferred to the asymmetries of leptons (or quarks) via the decays of the scalar fields. The AD mechanism also permits low temperature generation of baryon asymmetry, i.e., it may account for our baryonic universe without conflict to the gravitino problem [68]. We however need to fix the interactions of these scalar fields and inflaton, distributions for scalar fields, dynamics of inflation for an initial condition of AD baryogenesis, and so on to ensure successful lift-up of these scalar fields along the flat direction. Since realistic distributions for a lot of scalar fields remain a matter of research, we leave it as a subject for future work.

\section{Summary and discussion}

We have investigated the thermal leptogenesis in the $E_{6} \times \mathrm{U}(1)_{A}$ GUT model in which realistic quark and lepton masses and mixings are obtained and the doublet-triplet splitting problem is solved with natural assumption that all interactions including higher dimensional interactions are introduced with $O(1)$ coefficients. Each of three fundamental representations 27 includes two SM singlet fields, $S\left(\mathbf{1}^{\prime}\right)$ and $N_{R}^{c}(\mathbf{1})$, and these singlet fields play a role of RH neutrinos $N_{\alpha}(\alpha=1,2, \ldots, 6)$. One of the aim of this work is to show a sufficient lepton asymmetry is generated by the $\mathrm{CP}$ asymmetric decays of the lightest $\mathrm{RH}$ neutrino. In the model, Majorana masses of the $\mathrm{RH}$ neutrinos $M_{\alpha}$ and the neutrino Yukawa couplings $Y_{\alpha i}$ are determined by the $\mathrm{U}(1)_{A}$ symmetry. So we can calculate the lepton asymmetry, but unfortunately the naive calculation results in too small abundance of the lepton asymmetry. Actually, the lightest RH neutrino mass is around $6 \times 10^{7} \mathrm{GeV}$, which is smaller than the Ibarra's lower bound $10^{8-9} \mathrm{GeV}$. Moreover, the factor $K$ and the CP asymmetry $\epsilon_{N_{1}}^{\mathrm{SM}}$ are evaluated as $K \sim 40$ and $\epsilon_{N_{1}}^{\mathrm{SM}} \sim 5 \times 10^{-9}$. Therefore, the lepton asymmetry is washed out strongly in this scenario, and even with $K \sim 1$, the $\epsilon_{N_{1}}^{\mathrm{SM}}$ is too small to obtain the sufficient number of lepton asymmetry.

We have shown that a key ingredient for successful leptogenesis is the enhancement of RH neutrino masses. The model can include a large number of higher dimensional interactions, and these interaction terms yield additional Majorana masses after developing the VEVs of negatively $\mathrm{U}(1)_{A}$ charged fields. The enhancements of the RH neutrino masses enhance the CP asymmetry $\epsilon \propto M_{1}$ and make the decay parameter $K \propto 1 / M_{1}$ smaller to be most efficient value $K \sim 1$. How large enhancement factor is required for the sufficient leptogenesis? To answer this question, we have calculated the lepton asymmetry including the effects of SUSY and flavor in the final state of the CP asymmetric decay. The result is that the enhancement factor $16-17$ is sufficient for the successful leptogenesis. About 300 
mass terms are sufficient to obtain this enhancement factor, and this number looks not to be difficult to be obtained in the $E_{6}$ GUT model. It is important that such enhancement of the lightest $\mathrm{RH}$ neutrino mass does not change the neutrino physics at the low energy scale. This is because the $E_{6} \times \mathrm{U}(1)_{A}$ GUT has six RH neutrinos which induces the same order of the amplitude of all elements of the light neutrino mass matrix.

We have calculated the lepton asymmetry in the $E_{6} \times \mathrm{U}(1)_{A}$ model in following four cases: (i) non-SUSY+non-flavor (ii) non-SUSY+flavor (iii) SUSY+non-flavor (iv) SUSY+flavor. These calculations have shown that both the effects of lepton flavor and SUSY are important. It is known that in the strong washout regime lepton flavor effect becomes sizable, though SUSY contribution is not so large. We have shown that SUSY contribution becomes important even in the strong washout regime if lepton flavor effect is included. The essential point is that even in the strong regime $K^{\mathrm{SM}}>1$, the washout effects of the muon and/or the electron can become weak, and therefore these lepton number abundances become sizable.

Of course, the obtained result for the enhancement factor 16-17 $\left(M_{1} \sim 9 \times 10^{8} \mathrm{GeV}\right)$ for the sufficient leptogenesis is dependent on the various parameters and even on the $O(1)$ coefficients. For example, we have fixed the coefficient of $\epsilon_{N_{1}}^{\mathrm{SM}}$ in eq. (4.4) as two in our calculation. Since the final lepton asymmetry is proportional to this $\epsilon_{N_{1}}^{\mathrm{SM}}$ parameter, the dependence can be read from the figure 1. When the coefficient is one, the required enhancement factor becomes around $25\left(M_{1} \sim 1.4 \times 10^{9} \mathrm{GeV}\right)$, and therefore, $O(600)$ mass terms are needed. When the coefficient is four, it becomes around $10\left(M_{1} \sim 6 \times\right.$ $10^{8} \mathrm{GeV}$ ), which is required $O(100)$ mass terms. Therefore, we will not predict the mass of the lightest $\mathrm{RH}$ neutrino for sufficient leptogenesis, because it depends on the various parameters. An important thing is that the $E_{6} \times \mathrm{U}(1)_{A}$ GUT can explain the baryon asymmetry in the universe.

It is not plausible to produce the sufficient lepton number in the $\mathrm{SO}(10) \times \mathrm{U}(1)_{A}$ GUT [26] by the enhancement of the lightest RH neutrino. ${ }^{3}$ Since the number of the RH neutrinos is three in $\mathrm{SO}(10)$ model, the other two neutrinos must have the masses expected by the symmetry. However, the difference between the $\mathrm{U}(1)_{A}$ charges of the lightest $\mathrm{RH}$ neutrino and the second lightest RH neutrino mass terms is just two, and therefore, it is not reasonable to expect that the lightest $\mathrm{RH}$ neutrino has $O(10)$ times larger mass than that of the second lightest RH neutrino. However, since the $E_{6} \times \mathrm{U}(1)_{A}$ has six $\mathrm{RH}$ neutrinos and the difference between the $\mathrm{U}(1)_{A}$ charges of the lightest $\mathrm{RH}$ neutrino and the fifth lightest $\mathrm{RH}$ neutrino mass terms is six, it is plausible that the lightest $\mathrm{RH}$ neutrino has $O(100)$ times larger number of mass terms than that of the forth lightest $\mathrm{RH}$ neutrino. The observed baryon asymmetry in our universe may be an indirect signature of $E_{6}$ GUT.

\section{Acknowledgments}

This work was supported in part by the Grant-in-Aid for the Ministry of Education, Culture, Sports, Science, and Technology, Government of Japan, No. 15K05048 (N.M.) and No. 25003345 (M.Y.).

\footnotetext{
${ }^{3}$ There are various types of GUT scenarios wherein leptogenesis accounts for the baryon asymmetry [41, 69-84].
} 
Open Access. This article is distributed under the terms of the Creative Commons Attribution License (CC-BY 4.0), which permits any use, distribution and reproduction in any medium, provided the original author(s) and source are credited.

\section{References}

[1] H. Georgi and S.L. Glashow, Unity of All Elementary Particle Forces, Phys. Rev. Lett. 32 (1974) 438 [INSPIRE].

[2] E. Witten, Dynamical Breaking of Supersymmetry, Nucl. Phys. B 188 (1981) 513 [InSPIRE].

[3] S. Dimopoulos, S. Raby and F. Wilczek, Supersymmetry and the Scale of Unification, Phys. Rev. D 24 (1981) 1681 [InSPIRE].

[4] S. Dimopoulos and H. Georgi, Softly Broken Supersymmetry and SU(5), Nucl. Phys. B 193 (1981) 150 [INSPIRE].

[5] N. Sakai, Naturalness in Supersymmetric Guts, Z. Phys. C 11 (1981) 153 [INSPIRE].

[6] H. Fritzsch and P. Minkowski, Unified Interactions of Leptons and Hadrons, Annals Phys. 93 (1975) 193 [INSPIRE].

[7] F. Gursey, P. Ramond and P. Sikivie, A Universal Gauge Theory Model Based on E6, Phys. Lett. B 60 (1976) 177 [INSPIRE].

[8] Y. Achiman and B. Stech, Quark Lepton Symmetry and Mass Scales in an E6 Unified Gauge Model, Phys. Lett. B 77 (1978) 389 [InSPIRE].

[9] R. Barbieri and D.V. Nanopoulos, An Exceptional Model for Grand Unification, Phys. Lett. B 91 (1980) 369 [INSPIRE].

[10] T. Kugo and J. Sato, Dynamical symmetry breaking in an $E_{6}$ GUT model, Prog. Theor. Phys. 91 (1994) 1217 [hep-ph/9402357] [INSPIRE].

[11] N. Irges, S. Lavignac and P. Ramond, Predictions from an anomalous U(1) model of Yukawa hierarchies, Phys. Rev. D 58 (1998) 035003 [hep-ph/9802334] [INSPIRE].

[12] M. Bando and T. Kugo, Neutrino masses in E 6 unification, Prog. Theor. Phys. 101 (1999) 1313 [hep-ph/9902204] [INSPIRE].

[13] M. Bando, T. Kugo and K. Yoshioka, Mass matrices in $E_{6}$ unification, Prog. Theor. Phys. 104 (2000) 211 [hep-ph/0003220] [INSPIRE].

[14] M. Bando and N. Maekawa, $E_{6}$ unification with b-large neutrino mixing, Prog. Theor. Phys. 106 (2001) 1255 [hep-ph/0109018] [INSPIRE].

[15] N. Maekawa, NonAbelian horizontal symmetry and anomalous U(1) symmetry for supersymmetric flavor problem, Phys. Lett. B 561 (2003) 273 [hep-ph/0212141] [INSPIRE].

[16] N. Maekawa, E $E_{6}$ unification, large neutrino mixings and SUSY flavor problem, Prog. Theor. Phys. 112 (2004) 639 [hep-ph/0402224] [InSPIRE].

[17] M. Ishiduki, S.G. Kim, N. Maekawa and K. Sakurai, CEDM constraints on modified sfermion universality and spontaneous CP-violation, Prog. Theor. Phys. 122 (2009) 659 [arXiv: 0901.3400] [INSPIRE].

[18] M. Ishiduki, S.G. Kim, N. Maekawa and K. Sakurai, Spontaneous CP-violation in $E_{6}$ SUSY GUT with SU(2) flavor and anomalous U(1) symmetries, Phys. Rev. D 80 (2009) 115011 [Erratum ibid. D 81 (2010) 039901] [arXiv:0910.1336] [inSPIRE]. 
[19] H. Kawase and N. Maekawa, Flavor structure of $E_{6}$ GUT models, Prog. Theor. Phys. 123 (2010) 941 [arXiv: 1005.1049] [INSPIRE].

[20] N. Maekawa and K. Takayama, Neutrino properties in $E_{6} \times \mathrm{SU}(2)_{F} S U S Y$ GUT with spontaneous CP-violation, Phys. Rev. D 85 (2012) 095015 [arXiv:1202.5816] [INSPIRE].

[21] L. Randall and C. Csáki, The Doublet-triplet splitting problem and Higgses as pseudoGoldstone bosons, hep-ph/9508208 [INSPIRE].

[22] E. Witten, Some Properties of O(32) Superstrings, Phys. Lett. B 149 (1984) 351 [InSPIRE].

[23] M. Dine, N. Seiberg and E. Witten, Fayet-Iliopoulos Terms in String Theory, Nucl. Phys. B 289 (1987) 589 [INSPIRE].

[24] J.J. Atick, L.J. Dixon and A. Sen, String Calculation of Fayet-Iliopoulos d Terms in Arbitrary Supersymmetric Compactifications, Nucl. Phys. B 292 (1987) 109 [INSPIRE].

[25] M. Dine, I. Ichinose and N. Seiberg, F terms and d Terms in String Theory, Nucl. Phys. B 293 (1987) 253 [INSPIRE]

[26] N. Maekawa, Neutrino masses, anomalous U(1) gauge symmetry and doublet-triplet splitting, Prog. Theor. Phys. 106 (2001) 401 [hep-ph/0104200] [INSPIRE].

[27] N. Maekawa and T. Yamashita, $E_{6}$ unification, doublet triplet splitting and anomalous $\mathrm{U}(1)_{A}$ symmetry, Prog. Theor. Phys. 107 (2002) 1201 [hep-ph/0202050] [INSPIRE].

[28] N. Maekawa and T. Yamashita, Simple $E_{6}$ unification with anomalous $\mathrm{U}(1)_{A}$ symmetry, Prog. Theor. Phys. 110 (2003) 93 [hep-ph/0303207] [INSPIRE].

[29] N. Maekawa, Gauge coupling unification with anomalous $\mathrm{U}(1)_{A}$ gauge symmetry, Prog. Theor. Phys. 107 (2002) 597 [hep-ph/0111205] [INSPIRE].

[30] N. Maekawa and T. Yamashita, Gauge coupling unification in GUT with anomalous U(1) symmetry, Phys. Rev. Lett. 90 (2003) 121801 [hep-ph/0209217] [INSPIRE].

[31] C.D. Froggatt and H.B. Nielsen, Hierarchy of Quark Masses, Cabibbo Angles and CP-violation, Nucl. Phys. B 147 (1979) 277 [INSPIRE].

[32] L.E. Ibáñez and G.G. Ross, Fermion masses and mixing angles from gauge symmetries, Phys. Lett. B 332 (1994) 100 [hep-ph/9403338] [INSPIRE].

[33] M. Fukugita and T. Yanagida, Baryogenesis Without Grand Unification, Phys. Lett. B 174 (1986) 45 [INSPIRE].

[34] S. Davidson and A. Ibarra, A lower bound on the right-handed neutrino mass from leptogenesis, Phys. Lett. B 535 (2002) 25 [hep-ph/0202239] [INSPIRE].

[35] T2K collaboration, K. Abe et al., Indication of Electron Neutrino Appearance from an Accelerator-produced Off-axis Muon Neutrino Beam, Phys. Rev. Lett. 107 (2011) 041801 [arXiv:1106.2822] [INSPIRE].

[36] Double CHOOZ collaboration, Y. Abe et al., Indication for the disappearance of reactor electron antineutrinos in the Double CHOOZ experiment, Phys. Rev. Lett. 108 (2012) 131801 [arXiv: 1112.6353] [INSPIRE].

[37] DAYA BAY collaboration, F.P. An et al., Observation of electron-antineutrino disappearance at Daya Bay, Phys. Rev. Lett. 108 (2012) 171803 [arXiv:1203.1669] [INSPIRE].

[38] DAYA BAY collaboration, F.P. An et al., Improved Measurement of Electron Antineutrino Disappearance at Daya Bay, Chin. Phys. C 37 (2013) 011001 [arXiv:1210.6327] [InSPIRE]. 
[39] RENO collaboration, J.K. Ahn et al., Observation of Reactor Electron Antineutrino Disappearance in the RENO Experiment, Phys. Rev. Lett. 108 (2012) 191802 [arXiv: 1204.0626] [INSPIRE].

[40] V.A. Kuzmin, V.A. Rubakov and M.E. Shaposhnikov, On the Anomalous Electroweak Baryon Number Nonconservation in the Early Universe, Phys. Lett. B 155 (1985) 36 [INSPIRE].

[41] M. Plümacher, Baryon asymmetry, neutrino mixing and supersymmetric $\mathrm{SO}(10)$ unification, Nucl. Phys. B 530 (1998) 207 [hep-ph/9704231] [INSPIRE].

[42] L. Covi, E. Roulet and F. Vissani, CP violating decays in leptogenesis scenarios, Phys. Lett. B 384 (1996) 169 [hep-ph/9605319] [INSPIRE].

[43] E.W. Kolb and M.S. Turner, The Early Universe, Addison-Wesley, Redwood City, (1990).

[44] J.A. Harvey and M.S. Turner, Cosmological baryon and lepton number in the presence of electroweak fermion number violation, Phys. Rev. D 42 (1990) 3344 [InSPIRE].

[45] Particle Data Group collaboration, K.A. Olive et al., Review of Particle Physics, Chin. Phys. C 38 (2014) 090001 [inSPIRE].

[46] E. Nardi, Y. Nir, J. Racker and E. Roulet, On Higgs and sphaleron effects during the leptogenesis era, JHEP 01 (2006) 068 [hep-ph/0512052] [INSPIRE].

[47] E. Nardi, Y. Nir, E. Roulet and J. Racker, The importance of flavor in leptogenesis, JHEP 01 (2006) 164 [hep-ph/0601084] [INSPIRE].

[48] S. Davidson, E. Nardi and Y. Nir, Leptogenesis, Phys. Rept. 466 (2008) 105 [arXiv:0802.2962] [INSPIRE].

[49] M. Laine and M.E. Shaposhnikov, A remark on sphaleron erasure of baryon asymmetry, Phys. Rev. D 61 (2000) 117302 [hep-ph/9911473] [InSPIRE].

[50] D.J.H. Chung, B. Garbrecht, M. Ramsey-Musolf and S. Tulin, Supergauge interactions and electroweak baryogenesis, JHEP 12 (2009) 067 [arXiv:0908.2187] [INSPIRE].

[51] C.S. Fong, M.C. Gonzalez-Garcia, E. Nardi and J. Racker, Supersymmetric Leptogenesis, JCAP 12 (2010) 013 [arXiv: 1009.0003] [INSPIRE].

[52] S. Weinberg, Cosmological Constraints on the Scale of Supersymmetry Breaking, Phys. Rev. Lett. 48 (1982) 1303 [INSPIRE].

[53] M. Yu. Khlopov and A.D. Linde, Is It Easy to Save the Gravitino?, Phys. Lett. B 138 (1984) 265 [INSPIRE].

[54] J.R. Ellis, J.E. Kim and D.V. Nanopoulos, Cosmological Gravitino Regeneration and Decay, Phys. Lett. B 145 (1984) 181 [INSPIRE].

[55] M.H. Reno and D. Seckel, Primordial Nucleosynthesis: The Effects of Injecting Hadrons, Phys. Rev. D 37 (1988) 3441 [inSPIRE].

[56] M. Kawasaki and T. Moroi, Gravitino production in the inflationary universe and the effects on big bang nucleosynthesis, Prog. Theor. Phys. 93 (1995) 879 [hep-ph/9403364] [INSPIRE].

[57] K. Kohri, Primordial nucleosynthesis and hadronic decay of a massive particle with a relatively short lifetime, Phys. Rev. D 64 (2001) 043515 [astro-ph/0103411] [INSPIRE].

[58] M. Kawasaki, K. Kohri and T. Moroi, Big-Bang nucleosynthesis and hadronic decay of long-lived massive particles, Phys. Rev. D 71 (2005) 083502 [astro-ph/0408426] [INSPIRE]. 
[59] M. Kawasaki, K. Kohri, T. Moroi and A. Yotsuyanagi, Big-Bang Nucleosynthesis and Gravitino, Phys. Rev. D 78 (2008) 065011 [arXiv:0804.3745] [InSPIRE].

[60] M.A. Luty and R. Sundrum, Supersymmetry breaking and composite extra dimensions, Phys. Rev. D 65 (2002) 066004 [hep-th/0105137] [INSPIRE].

[61] M. Luty and R. Sundrum, Anomaly mediated supersymmetry breaking in four-dimensions, naturally, Phys. Rev. D 67 (2003) 045007 [hep-th/0111231] [INSPIRE].

[62] M. Ibe, R. Kitano, H. Murayama and T. Yanagida, Viable supersymmetry and leptogenesis with anomaly mediation, Phys. Rev. D 70 (2004) 075012 [hep-ph/0403198] [INSPIRE].

[63] K. Kumekawa, T. Moroi and T. Yanagida, Flat potential for inflaton with a discrete $R$ invariance in supergravity, Prog. Theor. Phys. 92 (1994) 437 [hep-ph/9405337] [InSPIRE].

[64] G. Lazarides, Leptogenesis in supersymmetric hybrid inflation, Springer Tracts Mod. Phys. 163 (2000) 227 [hep-ph/9904428] [INSPIRE].

[65] G.F. Giudice, M. Peloso, A. Riotto and I. Tkachev, Production of massive fermions at preheating and leptogenesis, JHEP 08 (1999) 014 [hep-ph/9905242] [INSPIRE].

[66] T. Asaka, K. Hamaguchi, M. Kawasaki and T. Yanagida, Leptogenesis in inflaton decay, Phys. Lett. B 464 (1999) 12 [hep-ph/9906366] [INSPIRE].

[67] I. Affleck and M. Dine, A New Mechanism for Baryogenesis, Nucl. Phys. B 249 (1985) 361 [INSPIRE].

[68] M. Dine, L. Randall and S.D. Thomas, Baryogenesis from flat directions of the supersymmetric standard model, Nucl. Phys. B 458 (1996) 291 [hep-ph/9507453] [INSPIRE].

[69] E. Nezri and J. Orloff, Neutrino oscillations versus leptogenesis in $\mathrm{SO}(10)$ models, JHEP 04 (2003) 020 [hep-ph/0004227] [inSPIRE].

[70] T. Hambye, E. Ma, M. Raidal and U. Sarkar, Allowable low-energy $E_{6}$ subgroups from leptogenesis, Phys. Lett. B 512 (2001) 373 [hep-ph/0011197] [INSPIRE].

[71] F. Buccella, D. Falcone and F. Tramontano, Baryogenesis via leptogenesis in $\mathrm{SO}(10)$ models, Phys. Lett. B 524 (2002) 241 [hep-ph/0108172] [INSPIRE].

[72] T. Asaka, Lopsided mass matrices and leptogenesis in $\mathrm{SO}(10)$ GUT, Phys. Lett. B 562 (2003) 291 [hep-ph/0304124] [INSPIRE].

[73] M.-C. Chen and K.T. Mahanthappa, Lepton flavor violating decays, soft leptogenesis and SUSY SO(10), Phys. Rev. D 70 (2004) 113013 [hep-ph/0409096] [INSPIRE].

[74] X.-d. Ji, Y.-c. Li, R.N. Mohapatra, S. Nasri and Y. Zhang, Leptogenesis in Realistic SO(10) Models, Phys. Lett. B 651 (2007) 195 [hep-ph/0605088] [INSPIRE].

[75] E.J. Chun and L. Velasco-Sevilla, $\mathrm{SO}(10)$ unified models and soft leptogenesis, JHEP 08 (2007) 075 [hep-ph/0702039] [INSPIRE].

[76] J.C. Romao, M.A. Tortola, M. Hirsch and J.W.F. Valle, Fermion masses, Leptogenesis and Supersymmetric SO(10) Unification, Phys. Rev. D 77 (2008) 055002 [arXiv:0707.2942] [INSPIRE].

[77] S. Blanchet and P. Fileviez Perez, Baryogenesis via Leptogenesis in Adjoint SU(5), JCAP 08 (2008) 037 [arXiv: 0807.3740] [INSPIRE].

[78] S.K. Majee, M.K. Parida and A. Raychaudhuri, Neutrino mass and low-scale leptogenesis in a testable SUSY SO(10) model, Phys. Lett. B 668 (2008) 299 [arXiv:0807.3959] [InSPIRE]. 
[79] A. Abada, P. Hosteins, F.-X. Josse-Michaux and S. Lavignac, Successful Leptogenesis in $\mathrm{SO}(10)$ Unification with a Left-Right Symmetric Seesaw Mechanism, Nucl. Phys. B 809 (2009) 183 [arXiv:0808.2058] [INSPIRE].

[80] P. Di Bari and A. Riotto, Testing SO(10)-inspired leptogenesis with low energy neutrino experiments, JCAP 04 (2011) 037 [arXiv:1012.2343] [INSPIRE].

[81] K. Kannike and D.V. Zhuridov, New Solution for Neutrino Masses and Leptogenesis in Adjoint SU(5), JHEP 07 (2011) 102 [arXiv: 1105.4546] [INSPIRE].

[82] F. Buccella, D. Falcone, C.S. Fong, E. Nardi and G. Ricciardi, Squeezing out predictions with leptogenesis from SO(10), Phys. Rev. D 86 (2012) 035012 [arXiv:1203.0829] [INSPIRE].

[83] P. Di Bari, L. Marzola and M. Re Fiorentin, Decrypting SO(10)-inspired leptogenesis, Nucl. Phys. B 893 (2015) 122 [arXiv: 1411.5478] [INSPIRE].

[84] C.S. Fong, D. Meloni, A. Meroni and E. Nardi, Leptogenesis in SO(10), JHEP 01 (2015) 111 [arXiv: 1412.4776] [INSPIRE]. 